\title{
Assessing structural, free-charge carrier, and phonon properties of mixed-phase epitaxial films: The case of InN
}

Mengyao Xie, M. Schubert, Jun Lu, Per O A Persson, Vallery Stanishev, Ching-Lien Hsiao,

L. C. Chen, W. J. Schaff and Vanya Darakchieva

\section{Linköping University Post Print}

\section{Tweet}

N.B.: When citing this work, cite the original article.

Original Publication:

Mengyao Xie, M. Schubert, Jun Lu, Per O A Persson, Vallery Stanishev, Ching-Lien Hsiao, L. C. Chen, W. J. Schaff and Vanya Darakchieva, Assessing structural, free-charge carrier, and phonon properties of mixed-phase epitaxial films: The case of InN, 2014, Physical Review B. Condensed Matter and Materials Physics, (90), 19, 195306.

http://dx.doi.org/10.1103/PhysRevB.90.195306

Copyright: American Physical Society http://www.aps.org/

Postprint available at: Linköping University Electronic Press http://urn.kb.se/resolve?urn=urn:nbn:se:liu:diva-113012 


\title{
Assessing structural, free-charge carrier, and phonon properties of mixed-phase epitaxial films: The case of $\mathrm{InN}$
}

\author{
M.-Y. Xie, ${ }^{1}$ M. Schubert, ${ }^{2}$ J. Lu, ${ }^{1}$ P. O. Å. Persson, ${ }^{1}$ V. Stanishev, ${ }^{1}$ C. L. Hsiao, ${ }^{1}$ L. C. Chen,${ }^{3}$ W. J. Schaff, ${ }^{4}$ and V. Darakchieva ${ }^{1}$ \\ ${ }^{1}$ Department of Physics, Chemistry and Biology, IFM, Linköping University, SE-581 83 Linköping, Sweden \\ ${ }^{2}$ Department of Electrical and Computer Engineering, University of Nebraska, Lincoln, Nebraska 68588, USA \\ ${ }^{3}$ Center for Condensed Matter Sciences, National Taiwan University, Taipei 106, Taiwan \\ ${ }^{4}$ Department of Electrical and Computer Engineering, Cornell University, Ithaca, New York 14853, USA \\ (Received 5 February 2014; revised manuscript received 27 October 2014; published 14 November 2014)
}

\begin{abstract}
We develop and discuss appropriate methods based on x-ray diffraction and generalized infrared spectroscopic ellipsometry to identify wurtizte and zinc-blende polymorphs, and quantify their volume fractions in mixed-phase epitaxial films taking $\mathrm{InN}$ as an example. The spectral signatures occurring in the azimuth polarization (Müller matrix) maps of mixed-phase epitaxial $\mathrm{InN}$ films are discussed and explained in view of polymorphism (zinc-blende versus wurtzite), volume fraction of different polymorphs and their crystallographic orientation, and azimuth angle. A comprehensive study of the structural, phonon and free electron properties of zinc-blende InN films containing inclusions of wurtzite $\mathrm{InN}$ is also presented. Thorough analysis on the formation of the zinc-blende and wurtzite phases is given and the structural evolution with film thickness is discussed in detail. The phonon properties of the two phases are determined and discussed together with the determination of the bulk free-charge carrier concentration, and electron accumulation at the mixed-phase $\operatorname{InN}$ film surfaces.
\end{abstract}

PACS number(s): 81.05.Ea, 78.30.Fs, 63.20.-e, 68.37.Og

\section{INTRODUCTION}

Polymorphism is ubiquitous to most materials independently of whether they occur naturally or are synthesized. Carbon, silicon carbide, and group-III-group-V compounds are among the notorious examples of technologically important materials exhibiting polymorphs that belong to the hexagonal and cubic crystal systems. The temperature, pressure, and chemical growth environment affect the crystallization process and can determine the resulting polymorphism. For example, diamond requires very high pressure to crystallize, while graphite forms at lower pressures. Many polymorphs are only stable within a certain range of conditions, and transitions from one polymorph to another are possible depending on the growth or post-growth conditions. Some polymorphic transitions involve extensive internal rearrangement and reconstruction of the crystal and subsequently require significantly more energy. However, in some instances, only a change in the stacking sequence can lead to the formation of a different polymorph. Consequently, the coexistence of two (or more) polymorphs in the crystal or thin film grown under nonequilibrium conditions may be expected. Developing nondestructive methods and approaches to study the properties of thin films containing different polymorphs is indispensable for appropriate analysis of their structural, electrical, optical and electronic properties, for example.

The group-III nitride family, InN, GaN, AlN, and their alloys is an intriguing example of a material system that exhibits polymorphism and attracts substantial research interest due to their unique properties and importance for optoelectronic and electronic devices [1-6]. The group-III nitrides crystalize in two polymorhs, the wurtzite (WZ) structure with space group $C_{6 v}^{4}\left(P 6_{3} m c\right)$ and the zinc-blende (ZB) structure with $T_{d}^{2}(F \overline{4} 3 m)$ symmetry. In both structures, each atom is tetrahedrally surrounded by atoms of the other element. The two polymorphs differ only by their stacking sequence, which is $\mathrm{ABAB}$ along the $\mathrm{WZ}\langle 0001\rangle$ and $\mathrm{ABCABC}$ along the $\mathrm{ZB}$ $\langle 111\rangle$, respectively (see Fig. 1). The WZ crystal structure is the thermodynamically stable configuration of these compounds. However, the ZB structure has only a slightly higher structural energy due to the same next neighbor configuration and the ionic character of the bonds. Consequently, group-III nitrides can be grown in the metastable $\mathrm{ZB}$ phase using growth techniques operating under conditions that are far from equilibrium.

ZB group-III nitrides have many advantageous properties over their WZ counterparts, e.g., smaller electron and hole effective mass parameters, a higher saturated electron drift velocity, and the possibility to produce laser facets by simple cleavage [7]. A strong built-in electrostatic field is present in WZ nitride heterostructures grown along the polar direction due to the spontaneous and piezoelectric polarizations. As a result, undesirable red-shift of the emission peaks and a reduction of the emission efficiency occur in light emitting devices containing WZ quantum well or quantum dot active regions. In contrast, for the ZB structure with 001 orientation, the spontaneous and piezoelectric polarizations are zero, due to its equal cation-to-anion bonds. Thus ZB group-III nitrides with 001 orientation offer a direct way to eliminate polarization effects which inherently limit the performance of $\mathrm{WZ}$ polar optoelectronic devices. ZB InN has the lowest band gap, the smallest effective mass and the highest electron mobility among group-III nitrides, and the widest phononic band gap and narrowest optical phonon band width among all group-IIIgroup- $\mathrm{V}$ semiconductors [8]. These unique properties make $\mathrm{ZB}$ InN a very good candidate for solar cell and high-speed electronics applications, for example.

Using molecular beam epitaxy (MBE), known as a noneqilibrium growth method, $\mathrm{ZB} \mathrm{GaN}, \mathrm{AlGaN}$, and Garich InGaN with good crystalline quality have been grown [9-12]. The key parameters to achieve ZB films with high 

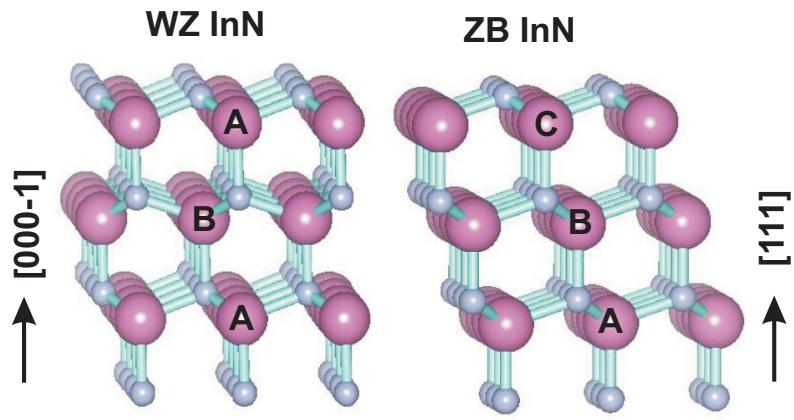

FIG. 1. (Color online) Stacking sequence of the ZB and WZ InN polymorhs.

phase purity are the choice of substrate and the growth temperature. Sometimes, undesirable formation of ZB phase in WZ group-III nitride films occurs, e.g., when doped with high concentrations of $\mathrm{Mg}$ to achieve $p$-type conductivity $[13,14]$.

In contrast to Ga-rich $\mathrm{ZB} \mathrm{Ga}(\mathrm{In}, \mathrm{Al}) \mathrm{N}$, which have been extensively studied, the basic parameters of $\mathrm{ZB}$ InN are still under debate and the growth of pure-phase $\mathrm{ZB} \mathrm{InN}$ has not been reported yet $[15,16]$. Often, single scan $\mathrm{X}$-ray diffraction (XRD) measurements or direct comparison of the XRD intensities of $\mathrm{WZ}$ and $\mathrm{ZB}$ InN peaks is used to conclude on the phase purity of ZB InN (001) epitaxial films [17]. However, it has been shown that WZ (0001) can be easily formed on the $\mathrm{ZB}(111)$ planes due to stacking faults $[15,18]$. This results in the growth of $\mathrm{WZ} \mathrm{InN}$ inclusions with the (1011) plane being almost parallel to the sample surface. Due to the small tilt of this WZ InN semipolar plane, its XRD peak can not be detected in single XRD scans in Bragg-Brentano geometry. In reciprocal space mapping (RSM), the WZ phase inclusion may remain hidden depending on the azimuth angle [19]. Furthermore, a direct comparison of diffraction intensities of $\mathrm{WZ}(10 \overline{1} 1)$ and $\mathrm{ZB}(002)$ peaks can result in significant underestimation of the WZ phase content due to the much higher structural factor of the ZB InN (002) planes. Therefore identification of the different polymorphs in InN (and other group-III nitrides) and proper quantification of their volume ratio is very important for a correct determination of the fundamental properties and parameters of the ZB phase, as well as for proper evaluation of mixed-phase group-III nitride epitaxial films. In this work, we develop and demonstrate nondestructive methods based on XRD and generalized infrared spectroscopic ellipsometry (GIRSE) to determine the phase ratio and study the structural, free-charge carrier and phonon properties of mixed-phase films containing ZB and WZ domains with distinct crystallographic pattern taking $\mathrm{InN}$ as an example.

\section{THEORY}

\section{A. Determination of phase ratio by XRD}

XRD involves probing a crystal with $\mathrm{x}$-ray radiation having a wavelength $\lambda$ close to the crystal lattice spacing. The $\mathrm{x}$ rays are scattered by the electron cloud surrounding each atom in the crystal. Constructive interference occurs between the scattered $\mathrm{x}$ rays when

$$
n \lambda=2 d \sin \theta,
$$

which is the well-known Bragg's law that relates the spacing $d$ between the planes of atoms from which diffraction is occurring to the angle $\theta$ at which the incident monochromatic beam must probe the plane to give constructive interference. By changing the angle of incidence, $\theta$, and detecting the signal at an angle of $2 \theta$ each set of crystal planes that fulfill the Bragg's law will produce a diffraction intensity. The integral intensity of a Bragg reflection is given by

$$
I_{h}=\operatorname{SCF} m_{h} T_{h} L_{p}\left|F_{h}\right|^{2} A_{\theta 2 \theta}(t) \frac{V}{V_{\mathrm{uc}}^{2}},
$$

where SCF is a scaling factor related to the specific instrument settings (scan velocity, slit width, etc.), $m_{h}$ is the multiplicity of the $h$ th Bragg reflection, $T_{h}$ is the texture factor of the $h$ th reflection, $L_{p}$ is the Lorentz-polarization factor, $F_{h}$ is the structure factor, $A_{\theta 2 \theta}(t)$ is the absorption factor, $V$ is the irradiated crystal volume, and $V_{\mathrm{uc}}$ is the unit cell volume. $m_{h}$ and $T_{h}$ are 1 for single crystals. The Lorentz-polarization factor $L_{p}$ is expressed as

$$
L_{p}=\left(1+\cos ^{2} 2 \theta\right) / \sin ^{2} \theta,
$$

and the absorption factor $A_{\theta 2 \theta}(t)$ is given by

$$
A_{\theta 2 \theta}=1-\exp \left(\frac{-2 \mu t}{\sin \theta}\right),
$$

where $t$ is the film thickness, $\mu=\rho \mu_{m}$ is the linear attenuation coefficient, $\rho$ is the density, and $\mu_{m}$ is the absorption coefficient.

Let us consider a situation when the film consists of two polymorph domains, $\alpha$ and $\beta$, each with a single crystal orientation $h$ and $k$, respectively. The volume fraction of different polymorph domains can be obtained from the ratio of the integral intensities [see Eq. (2)] of the $h$ th reflection of polymorph $\alpha$ and the $k$ th reflection of polymorph $\beta$ :

$$
\frac{V^{\alpha}}{V^{\beta}}=\frac{I_{h}^{\alpha} L_{p}^{\beta}\left|F_{k}^{\beta}\right|^{2} A_{\theta 2 \theta}^{\beta}(t)\left(V_{\mathrm{uc}}^{\alpha}\right)^{2}}{I_{h}^{\beta} L_{p}^{\alpha}\left|F_{h}^{\alpha}\right|^{2} A_{\theta 2 \theta}^{\alpha}(t)\left(V_{\mathrm{uc}}^{\beta}\right)^{2}}
$$

and

$$
\sum_{i} V^{i}=100 \% .
$$

\section{B. Generalized ellipsometry}

For optically anisotropic materials, it is necessary to apply the generalized ellipsometry approach because coupling between $p$ (parallel to the plane of incidence) and $s$ (perpendicular to the plane of incidence) polarized incident electromagnetic plane wave components occurs upon reflection off the sample surface. For the samples discussed here, the optical axes of the corundum-structure sapphire and WZ structure InN sample constituents are optically anisotropic with uniaxial optical properties [20-23]. In the generalized ellipsometry formalism, the interaction of electromagnetic plane waves with layered samples is described within the Jones or Müller matrix formalism [24]. The Müller matrix is defined by arranging 
incident and exiting Stokes vectors into a matrix form:

$$
\left(\begin{array}{l}
S_{0} \\
S_{1} \\
S_{2} \\
S_{3}
\end{array}\right)_{\text {output }}=\left(\begin{array}{llll}
M_{11} & M_{12} & M_{13} & M_{14} \\
M_{12} & M_{22} & M_{23} & M_{24} \\
M_{13} & M_{32} & M_{33} & M_{34} \\
M_{14} & M_{42} & M_{43} & M_{44}
\end{array}\right)\left(\begin{array}{c}
S_{0} \\
S_{1} \\
S_{2} \\
S_{3}
\end{array}\right)_{\text {input }}
$$

with $S_{0}=I_{p}+I_{s}, \quad S_{1}=I_{p}-I_{s}, \quad S_{2}=I_{45}-I_{-45}, \quad S_{3}=$ $I_{\sigma+}-I_{\sigma-}$, where $I_{p}, I_{s}, I_{45}, I_{-45}, I_{\sigma+}$, and $I_{\sigma-}$ denote the intensities for the $p-, s-,+45^{\circ},-45^{\circ}$, right-, and left-handed circularly polarized light components, respectively [25].

\section{Ellipsometry model dielectric function and data analysis}

The ellipsometry data are analyzed using a stratified layer model including the sapphire substrate and the $\mathrm{InN}$ layer. The InN layer is considered to be composed of bulk region and surface layer with different free electron concentration according to experimental findings of electron accumulation occurring at InN surfaces [23,26-28]. The light propagation within the entire sample stack is calculated by applying a $4 \times 4$ matrix algorithm for multilayer systems assuming plane parallel interfaces. In order to reduce parameter correlation, data measured from the same sample obtained at multiple angles of incidence and multiple sample azimuth angles are analyzed simultaneously. A regression analysis (Levenberg-Marquardt algorithm) is performed, where the model parameters are varied until calculated and experimental data match as close as possible (best-match model). This is done by minimizing the mean square error $\left(\chi^{2}\right)$ function, which is weighed to estimated experimental errors $(\sigma)$ determined by the instrument for each data point [29].

For necessary and convenient reduction of unknown parameters, analytical descriptions of the dielectric function components of each sample constituent (substrate, InN layer) are needed. For the substrate, the material is considered ideally as single-crystalline and one tensor is sufficient to render the optical properties. The infrared optical properties and tensor description of sapphire follows that reported previously [30], and which is not altered here.

For ZB InN, which is isotropic, the dielectric function tensor is diagonal, and its main value is equal to the isotropic dielectric function, $\varepsilon_{\mathrm{ZB}}$

$$
\varepsilon_{\mathrm{ZB}}=\operatorname{diag}\left(\varepsilon_{\mathrm{is}}, \varepsilon_{\mathrm{is}}, \varepsilon_{\mathrm{is}}\right),
$$

where "diag" indicates the diagonal matrix. The wavelength dependence of the dielectric function in the spectral vicinity of a polar lattice resonance is commonly described by a harmonic Lorentzian oscillator, which may be represented in fractional decomposition form:

$$
\varepsilon_{\mathrm{is}}=\varepsilon_{\infty} \frac{\omega_{\mathrm{LO}}^{2}-\omega^{2}-\mathrm{i} \omega \gamma}{\omega_{\mathrm{TO}}^{2}-\omega^{2}-\mathrm{i} \omega \gamma},
$$

where $\omega_{\mathrm{TO}}, \omega_{\mathrm{LO}}$, and $\gamma$ are transverse optical (TO), longitudinal optical (LO) frequencies, and broadening parameters, respectively, and $\varepsilon_{\infty}$ is the parameter for the high-frequency dielectric constant.

For WZ InN, which is uniaxial, the dielectric function tensor is diagonal, and its main values differ for polarizabilities parallel $(\|)$ and perpendicular $(\perp)$ to the lattice $c$ axis. For $c$-plane oriented (0001) InN, this tensor reads

$$
\varepsilon_{\mathrm{WZ},(0001)}=\operatorname{diag}\left(\varepsilon_{\perp}, \varepsilon_{\perp}, \varepsilon_{\|}\right) .
$$

The wavelength dependence of the dielectric function is described by the same harmonic Lorentzian oscillator approach:

$$
\varepsilon_{j}=\varepsilon_{\infty, j} \frac{\omega_{\mathrm{LO} j}^{2}-\omega^{2}-\mathrm{i} \omega \gamma_{j}}{\omega_{\mathrm{TO} j}^{2}-\omega^{2}-\mathrm{i} \omega \gamma_{j}} .
$$

The index " $j$ " stands for the dielectric function parameters for polarizability functions parallel $(\|)$ or perpendicular $(\perp)$ to the lattice $c$ axis. In addition to the contribution from polar phonons, the contribution from free-charge carriers to the InN model dielectric function needs to be accounted for. The classical Drude equation holds sufficiently for description of the contribution to $\varepsilon_{\text {Dr }}$ due to free charge carriers [29]:

$$
\varepsilon_{\mathrm{FC}}=-\frac{\mathrm{e}^{2} N_{\mathrm{s}}}{\tilde{\varepsilon}_{0} m_{\mathrm{eff}} \omega\left(\omega+\mathrm{i} \gamma_{\mathrm{p}}\right)},
$$

where $N_{\mathrm{s}}, m_{\text {eff }}$, and $\gamma_{\mathrm{p}}$ are the free-charge carrier volume density, effective mass, and plasma broadening parameters, respectively $\left(\tilde{\varepsilon}_{0}\right.$ is the vacuum permittivity, and $e$ is the amount of the electrical unit charge). The plasmon broadening parameter is related to the optical mobility parameter $\mu$ :

$$
\gamma_{\mathrm{p}}=\frac{e}{m_{\mathrm{eff}} \mu} .
$$

For simplicity, both the anisotropy of the WZ InN effective mass tensor as well as small differences between the isotropically averaged $\mathrm{WZ}$ InN effective mass value and the isotropic $\mathrm{ZB}$ InN effective mass value are assumed to vanish in this work. This assumption is based on the observation that the ellipsometry data analyzed and discussed below did not provide sufficient sensitivity to differentiate (a) between anisotropic effective mass parameters for the WZ and (b) between $\mathrm{ZB}$ and WZ InN contributions. Further work may be performed using the Optical Hall effect [31] where external magnetic fields provide increased sensitivity to free charge carrier parameters and which may permit determination of effective mass anisotropy [32-34]. The resulting free-charge carrier contribution is then assumed to be given by an isotropic tensor:

$$
\varepsilon_{\mathrm{Dr}}=\operatorname{diag}\left(\varepsilon_{\mathrm{FC}}, \varepsilon_{\mathrm{FC}}, \varepsilon_{\mathrm{FC}}\right) .
$$

\section{EXPERIMENT}

Two InN layers with intended ZB structure were grown by MBE on $r$-plane (1012) sapphire employing nitridation of the substrate prior to the growth (sample I) [17] and directly on the substrate (sample II) [15]. Sample I was grown at $400^{\circ} \mathrm{C}$, while sample II was grown at $470^{\circ} \mathrm{C}$. X-ray diffraction pole figure $(\mathrm{PF})$ and rocking curve (RC) measurements were performed using a D8Discover system with monochromatic $\mathrm{Cu} k_{\alpha_{1}}$ radiation. A Gobel mirror and an asymmetric two-bounce $\mathrm{Ge}(220)$ monochromator were used on the primary side and a position sensitive detector without any slits was employed on the secondary side. Reciprocal space maps (RSMs) at different azimuth angles were measured with a Philips triple axis diffractometer with a parabolic graded multilayer mirror on the primary side in triple-axis configuration. 
A focused ion-beam (FIB) FEI FIB2000 workstation was used to prepare cross-sectional specimens for transmission electron microscopy (TEM). TEM and high-resolution TEM (HRTEM) imaging was performed using a FEI Tecnai G2 TF20UT microscope operated at $200 \mathrm{kV}$. The vibrational properties and free-charge carrier properties were studied by room-temperature GIRSE. The GIRSE measurements were performed on a rotating compensator infrared ellipsometer (J. A. Woollam Co, Inc.) in the spectral range of $350-1500 \mathrm{~cm}^{-1}$ with a spectral resolution of $2 \mathrm{~cm}^{-1}$, and at $60^{\circ}$ and $70^{\circ}$ angles of incidence. The measurements at each angle of incidence were performed for four different sample azimuth angles $\varphi$ between the plane of incidence and the sapphire [0001] direction. The measurements are reported in terms of Müller matrix elements, which are normalized to element $M_{11}$.

\section{RESULTS AND DISCUSSION}

\section{A. Structural model}

Figure 2 shows the pole figures of selected $\mathrm{ZB}$ and $\mathrm{WZ}$ InN planes for the two InN samples. In both samples the presence of the ZB-InN (002) peak at a zero tilt is revealed. The four poles from the $\mathrm{ZB}$ InN (111) can also be detected at an inclination angle of $\approx 54^{\circ}$. However, the lattice spacings of the $\mathrm{ZB} \mathrm{InN} \mathrm{(111)} \mathrm{is} \mathrm{very} \mathrm{close} \mathrm{to} \mathrm{the} \mathrm{spacing} \mathrm{of} \mathrm{WZ}$ InN (0002) (see Fig. 1). Consequently, the diffraction from these two planes occur at very similar $2 \theta$ angles, and their contributions cannot be separated. We have recently reported that when WZ InN (0002) grows on ZB InN (111), the resulting semipolar WZ InN film has its (10ī1) plane slightly tilted with respect to the surface [18]. Indeed, four domains of WZ InN with $(10 \overline{1} 1)$ orientations, which are rotated by $90^{\circ}$ with respect to each other, are detected for the two samples studied here at an inclination angle of $7^{\circ}$ (see Fig. 2). These domains, hereafter named $\mathrm{A}, \mathrm{B}, \mathrm{C}$, and $\mathrm{D}$, occur at the same azimuth positions as the WZ InN (0002)/ZB InN (111) poles (Fig. 2) and indicate that non-negligible $\mathrm{WZ} \mathrm{InN}$ fraction has formed in both samples. The relative orientations of the $\mathrm{WZ}$ and $\mathrm{ZB}$ InN domains are schematically illustrated in Fig. 3. Only one

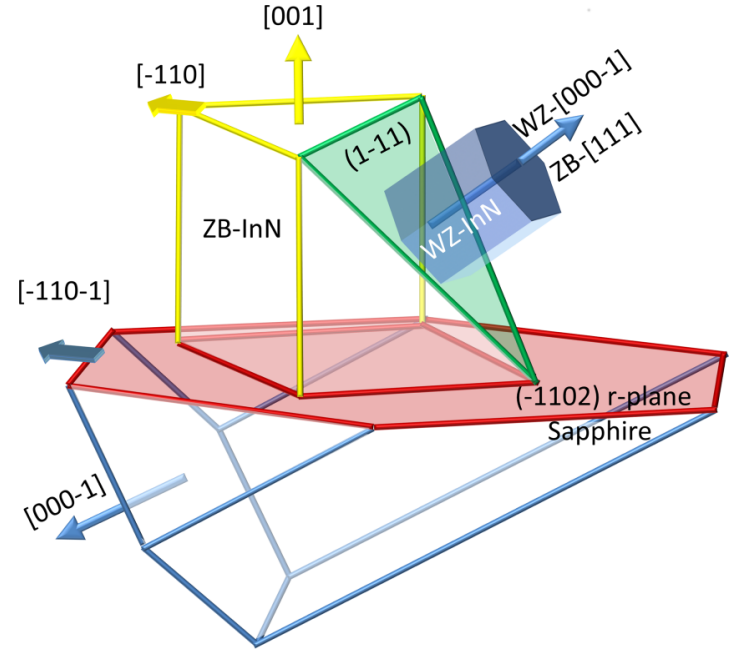

FIG. 3. (Color online) Schematic representation of the ZB and $\mathrm{WZ}$ polymorh orientations (not to scale). Only one of the four WZ InN domains forming on the $\mathrm{ZB} \mathrm{InN} \mathrm{(111)} \mathrm{is} \mathrm{shown.}$

of the four WZ InN domains formed at all four equivalent $\mathrm{ZB}$ InN (111) planes is shown in Fig. 3 for simplicity.

The WZ InN (1011) domains can also be identified in the reciprocal space maps around the $\mathrm{ZB}$ InN (002) reciprocal space point (see Fig. 4) if recorded in $1 \AA$ (or larger) range of the lateral scattering vector. At an azimuth angle of $1 \overline{10}$ the presence of WZ InN fraction with its [0001] direction parallel to the growth direction is also revealed for sample I [Fig. 4(a)]. Note that the WZ InN (0001) domain, although growing almost parallel to the sample surface, may remain hidden depending on the azimuth position. Note that a single $2 \theta-\omega$ scan (a vertical slice of the RSM through the zero lateral scattering vector) will only show the ZB (002) reflection and a small shoulder at the WZ (10 $\overline{1} 1)$ peak position. The WZ InN (10 $\overline{1} 1)$ is slightly tilted with respect to the sample surface and the tilt angle may vary depending on growth and nucleation condition [18].
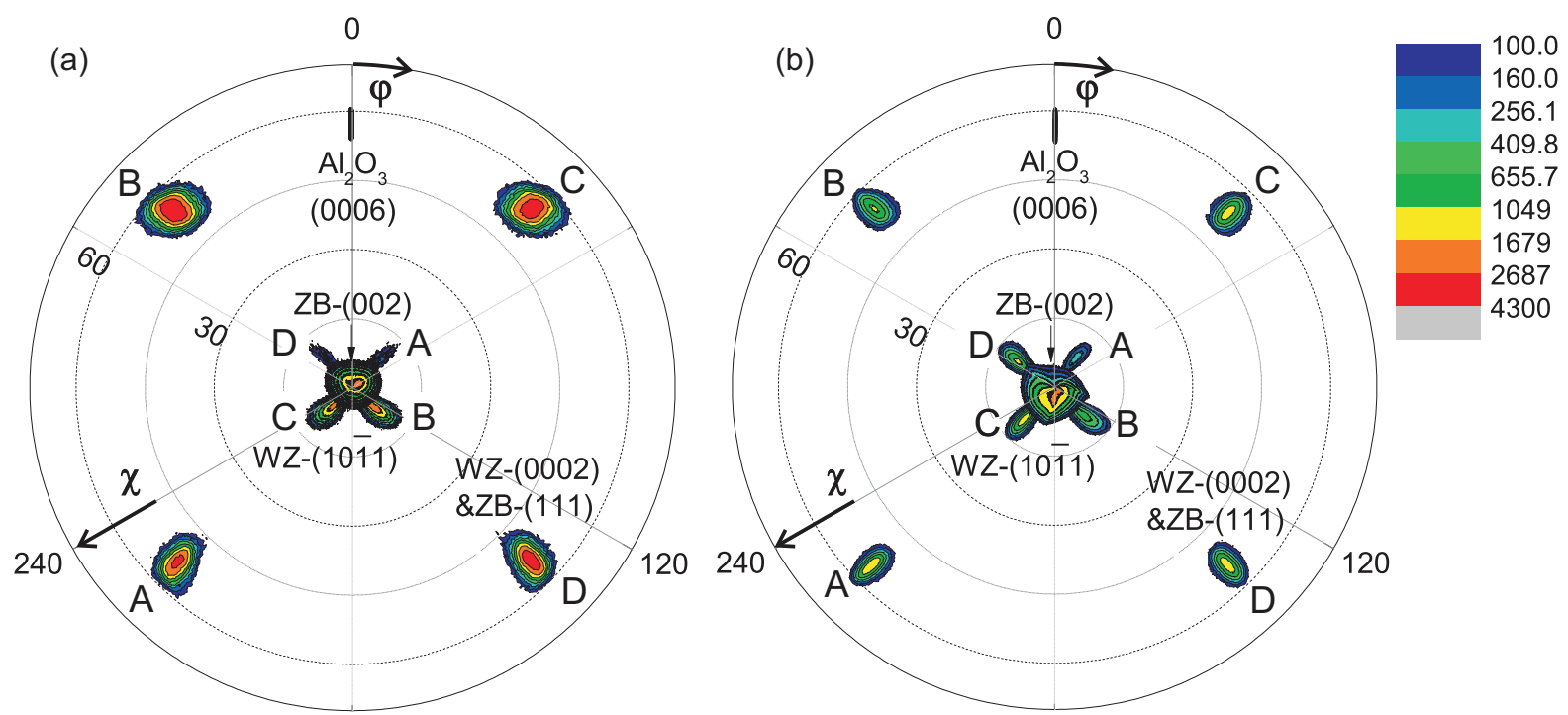

FIG. 2. (Color online) Stereographic projection of the pole figures for sample I (a) and sample II (b). 


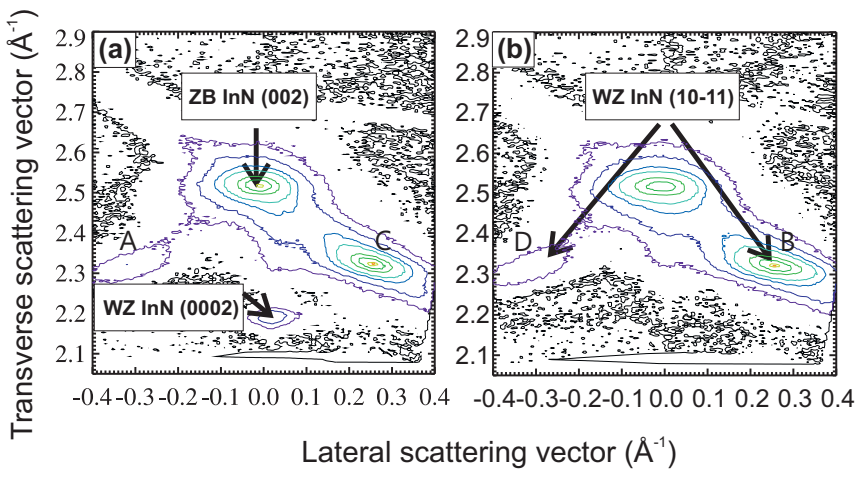

FIG. 4. (Color online) Reciprocal space maps around the ZB InN (002) reciprocal lattice point of sample I at (a) azimuth $1 \overline{1} 0$ and (b) azimuth 110 .

\section{B. Mixed-phase infrared dielectric function model}

In epitaxial films containing both $\mathrm{WZ}$ and $\mathrm{ZB}$ polymorphs, a concept for deriving the dielectric response of mixed-phase, multiple-domain thin films needs to be implemented. In this paper, we follow a linear tensor property averaging scheme. A similar linearization approach was previously used to calculate the effective dielectric tensor properties of highly textured polycrystalline thin films at long wavelengths [35]. In this concept, each domain is represented by its individual tensor as it would occur if the entire layer would be homogeneously filled by that domain. Then, the tensors of all occurring domains are added by weighing factors representing their volume fraction. In this linear homogenization approach, the effective dielectric tensor of the mixed-phase thin film is obtained as the sum of individual polytype domain contributions and the common contribution caused by free charge carriers $\varepsilon_{\mathrm{Dr}}$ :

$$
\varepsilon=\sum_{i} f_{i} \varepsilon_{i}+\varepsilon_{\mathrm{Dr}} .
$$

The summation goes over the individual polymorph domains, $i$.

For the InN films discussed here, according to the XRD analysis (see Figs. 2 and 4) the mixed-phase film consists of six domains: ZB with (002) orientation, four WZ domains A, $\mathrm{B}, \mathrm{C}$, and D with (1011) orientation, and in the case of sample I, also a WZ domain with (0001) orientation. The mixed-phase dielectric function model is constituted by contributions from all these domains:

$$
\begin{aligned}
\varepsilon= & f_{\mathrm{ZB}} \varepsilon_{\mathrm{ZB}}+f_{0001} \varepsilon_{0001}+f_{\mathrm{A}} \varepsilon_{\mathrm{A}}+f_{\mathrm{B}} \varepsilon_{\mathrm{B}} \\
& +f_{\mathrm{C}} \varepsilon_{\mathrm{C}}+f_{\mathrm{D}} \varepsilon_{\mathrm{D}} .
\end{aligned}
$$

The volume fraction parameters must obey

$$
1=f_{\mathrm{ZB}}+f_{0001}+f_{\mathrm{A}}+f_{\mathrm{B}}+f_{\mathrm{C}}+f_{\mathrm{D}} .
$$

The dielectric functions of the $\mathrm{ZB}$ and the WZ (0001) domains are described by Eqs. (8)-(11). The dielectric functions of the hexagonal domains $\mathrm{A}, \mathrm{B}, \mathrm{C}$, and $\mathrm{D}$ can be obtained by rotation of $\varepsilon_{\mathrm{WZ},(0001)}$ using Euler angles $\varphi$ and $\vartheta$,

$$
\varepsilon=\mathbf{B}(\vartheta) \mathbf{A}(\varphi) \varepsilon_{\mathrm{WZ},(0001)} \mathbf{A}(-\varphi) \mathbf{B}(-\vartheta),
$$

where Euler operations are defined as the usual right-handed rotations around the $z$ axis $(\mathbf{A}(\varphi))$ and $x$ axis $(\mathbf{B}(\vartheta))$.
According to structural analysis results (see Fig. 2), domains $\mathrm{A}\left(f_{\mathrm{A}} ; \varphi=\varphi_{0}\right), \mathrm{B}\left(f_{\mathrm{B}} ; \varphi=\varphi_{0}+\frac{\pi}{2}\right), \mathrm{C}\left(f_{\mathrm{C}} ; \varphi=\varphi_{0}+\pi\right)$, and $\mathrm{D}\left(f_{\mathrm{D}} ; \varphi=\varphi_{0}+\frac{3 \pi}{2}\right)$ differ in $\varphi$ by $\pi / 2$ each, while all domains share a common inclination $\vartheta=54^{\circ}$ (Fig. 3).

The effective dielectric tensor is obtained by adding the contribution caused by free-charge carriers $\varepsilon_{\mathrm{Dr}}$. The individual tensors representing the six different types of domains within the mixed-phase thin films are discussed below. The summation of all contributions leads to the following final tensor for the mixed-phase thin films:

$$
\varepsilon=\left(\begin{array}{lcc}
\varepsilon_{x x} & 0 & \varepsilon_{x z} \\
0 & \varepsilon_{y y} & \varepsilon_{y z} \\
\varepsilon_{z x} & \varepsilon_{z y} & \varepsilon_{z z}
\end{array}\right),
$$

with

$$
\begin{gathered}
\varepsilon_{x x}=\left(f_{\mathrm{A}}+f_{\mathrm{C}}+f_{0001}\right) \varepsilon_{\perp}+f_{\mathrm{ZB}} \varepsilon_{\mathrm{ZB}}+\varepsilon_{\mathrm{Dr}} \\
+\left(f_{\mathrm{B}}+f_{\mathrm{D}}\right)\left(\varepsilon_{\perp} \cos ^{2} \vartheta+\varepsilon_{\|} \sin ^{2} \vartheta\right) \\
\varepsilon_{y y}=\left(f_{\mathrm{B}}+f_{\mathrm{D}}+f_{0001}\right) \varepsilon_{\perp}+f_{\mathrm{ZB}} \varepsilon_{\mathrm{ZB}}+\varepsilon_{\mathrm{Dr}} \\
+\left(f_{\mathrm{A}}+f_{\mathrm{C}}\right)\left(\varepsilon_{\perp} \cos ^{2} \vartheta+\varepsilon_{\|} \sin ^{2} \vartheta\right) \\
\varepsilon_{z z}=f_{0001} \varepsilon_{\|}+f_{\mathrm{ZB}} \varepsilon_{\mathrm{ZB}}+\varepsilon_{\mathrm{Dr}} \\
+\left(f_{\mathrm{A}}+f_{\mathrm{B}}+f_{\mathrm{C}}+f_{\mathrm{D}}\right)\left(\varepsilon_{\perp} \cos ^{2} \vartheta+\varepsilon_{\|} \sin ^{2} \vartheta\right) \\
\varepsilon_{x z}=\varepsilon_{z x}=\left(f_{\mathrm{B}}-f_{\mathrm{D}}\right)\left(\varepsilon_{\|}-\varepsilon_{\perp}\right) \cos \vartheta \sin \vartheta \\
\varepsilon_{y z}=\varepsilon_{z y}=\left(f_{\mathrm{C}}-f_{\mathrm{A}}\right)\left(\varepsilon_{\|}-\varepsilon_{\perp}\right) \cos \vartheta \sin \vartheta
\end{gathered}
$$

Note that this tensor is symmetric, with no anisotropy between the $x$ and $y$ axes regardless of WZ domain fraction parameters, and that anisotropy between axes $x$ and $z$, and between axes $y$ and $z$ vanishes when oppositely located domains B and D, and $\mathrm{A}$ and $\mathrm{C}$ possess the same volume fractions, respectively. In particular, when $f_{\mathrm{A}}=f_{\mathrm{B}}=f_{\mathrm{C}}=f_{\mathrm{D}}$, the thin film optical response is that of a pseudo $c$-plane orientation, however, phonon modes signatures of both $\mathrm{WZ}$ and $\mathrm{ZB}$ domains will still occur in the ellipsometry spectra.

In order to demonstrate the sensitivity of the GIRSE to the presence of different domains and their volume fraction distributions, we have calculated selected Müller matrix data azimuthal maps for a mixed-phase InN film using the above discussed model and the best-match model parameters of sample II (see Table I). In Fig. 5, we exemplify representative $\mathrm{M}_{12}, \mathrm{M}_{13}$ and $\mathrm{M}_{43}$ azimuthal maps for wave numbers near the InN TO frequencies. We consider four cases of InN films

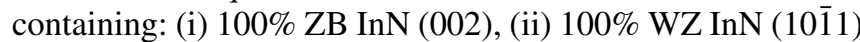
domain $\mathrm{A}$, (iii) $50 \% \mathrm{WZ} \mathrm{InN}(10 \overline{1} 1)$ domain $\mathrm{A}$ and $50 \% \mathrm{ZB}$ InN (002), and (iv) the best-match values for the volume fraction parameters of sample II obtained from the GIRSE analysis: $1 \% \mathrm{WZ}$ InN (10 $\overline{1} 1)$ domain A, 13\% WZ InN (1011) domain $\mathrm{B}, 44 \% \mathrm{WZ}$ InN (1011) domain C, 24\% WZ InN (1011) domain D, and 18\% ZB InN (002).

It is seen from Fig. 5 that distinct changes occur in the GIRSE data upon sample rotation depending on the polytype 

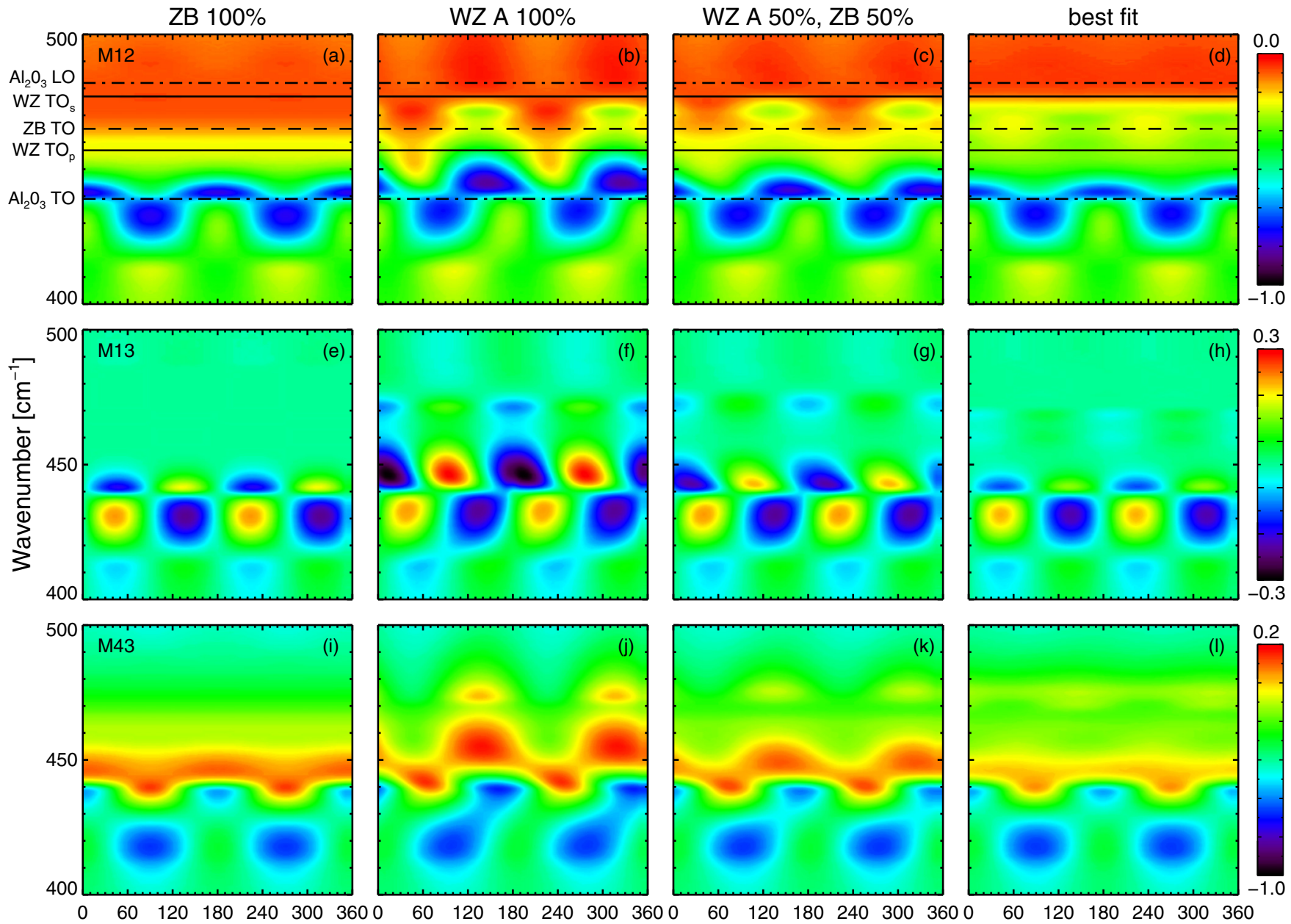

Azimuth angle $\varphi[\mathrm{deg}]$

FIG. 5. (Color online) Calculated Müller matrix data azimuth maps for elements $M_{12}$ (a)-(d), $M_{13}$ (e)-(h), and $M_{43}$ (i)-(l) over one full sample rotation for mixed-phase InN films on $r$-plane sapphire for wave numbers near the InN TO frequencies. The maps were simulated using our model approach described in this section and the best-match calculated model parameter values of sample II from Table I. Different volume fraction parameters are assumed for $\mathrm{WZ}$ and ZB domains as indicated above the respective maps: (a), (e), and (i) 100\% ZB (002) domain, (b), (f), and (j) 100\% WZ (1011) domain A, (c), (g), and (k) 50\% WZ (1011) domain A and 50\% ZB (002) domain, and (d), (h), and (l) the best-match model calculated parameter values for the volume fractions of sample II obtained from the GIRSE analysis: $1 \%$ WZ (10) 1 ) domain

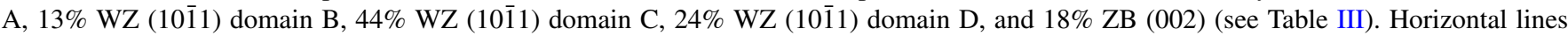
indicate wave numbers of sapphire phonon modes (dash-dotted lines), InN WZ TO phonon modes (solid lines) and InN ZB TO mode (dashed line).

volume fraction parameters. For instance, the low-frequency edge of the $\mathrm{InN}$ band of total reflection is marked by the $\mathrm{ZB}$ InN $\omega_{\mathrm{TO}, \mathrm{ZB}}$ frequency in the $\mathrm{M}_{12}$ azimuthal map for $\mathrm{ZB}$ volume fraction of $100 \%$ [dashed line in Fig. 5(a)]. The low-frequency edge position in this case is independent of the azimuth $\varphi$ (sample in-plane rotation) due to the isotropic nature of the $\mathrm{ZB}$ InN crystal structure. In contrast, when the InN film consists of $100 \% \mathrm{WZ}(10 \overline{1} 1)$ domain A, new features in the $\mathbf{M}_{12}$ azimuthal map occur in the vicinity of $\omega_{\mathrm{TO}, \perp}$ and $\omega_{\text {TO, }}$, which depend on the azimuth rotation [solid lines in Fig. 5(b)]. The total reflection band edge in $\mathrm{M}_{12}$ shifts from $\omega_{\mathrm{TO}, \|}$ at azimuth of $45^{\circ}$ to $\omega_{\mathrm{TO}, \perp}$ at azimuth of $135^{\circ}$. The $\omega_{\mathrm{TO}}, \|$ and $\omega_{\mathrm{TO}, \perp}$ are excited resonantly depending on $\varphi$ as the electric field parallel to the film interface senses lattice excitations with polarization parallel or perpendicular to the $\operatorname{InN} c$ axis, respectively. The specific azimuth pattern (horizontal slices through the maps at the WZ TO frequencies) is associated with the anisotropy of the InN wurtzite crystal structure and its $180^{\circ}$ basal plane symmetry. Note that there is a $45^{\circ}$ difference between the in-plane projections of the sapphire and $\mathrm{InN}$ $c$ axes (see Fig. 2). This also causes specific modifications in all maps in the vicinity of the sapphire TO phonon bands [dashed-dotted line in Figs. 5(a) and 5(b)]. Similar modifications are also observed in the $\mathbf{M}_{13}$ and $\mathbf{M}_{43}$ maps for the InN film containing $100 \% \mathrm{WZ}$ InN (1011) domain $\mathrm{A}$ in comparison to pure-phase $\mathrm{ZB}$ InN [see Figs. 5(e)-5(f) and 5(i)-5(j)]. In particular, the spectral features appearing in the $\mathrm{M}_{13}$ and $\mathrm{M}_{43}$ azimuthal maps above the indicated sapphire TO frequency [dashed-dotted line in Figs. 5(f) and 5(j)] are entirely caused by the presence of the WZ domain A.

In the case of a film containing $100 \%$ WZ (1011) domain B (not shown here), the spectral features in the GIRSE azimuthal 
TABLE I. Best-match ellipsometry data model parameters obtained for the mixed-phase InN samples studied in this work: film thickness, $t$, high-frequency dielectric function limit of $\mathrm{ZB}$ $\mathrm{InN}, \varepsilon_{\infty, \mathrm{ZB}}$, TO phonon frequency of $\mathrm{ZB} \mathrm{InN}, \omega_{\mathrm{TO}, \mathrm{ZB}}$, LO phonon frequency of $\mathrm{ZB}$ InN $\omega_{\mathrm{LO}, \mathrm{ZB}}$, phonon broadening parameter in $\mathrm{ZB}$ InN, $\gamma_{\mathrm{ZB}}, \mathrm{WZ}$ InN high-frequency dielectric function limit, $\varepsilon_{\infty, j}$, WZ InN TO phonon frequency, $\omega_{\mathrm{TO}, \mathrm{j}}$, WZ InN LO phonon frequency $\omega_{\mathrm{LO}, \|}$, WZ InN phonon broadening parameter, $\gamma_{\mathrm{j}}$ with $j=\|$ and $\perp$ for directions parallel and perpendicular to the $\mathrm{WZ} \operatorname{InN} c$ axis. $N$ and $\mu$ are the bulk free electron concentration and mobility, respectively, $N_{s}$ is the electron accumulation density at the surface. Error bars in parenthesis correspond to the $90 \%$ confidence intervals.

\begin{tabular}{lcc}
\hline \hline Parameter & Sample I & Sample II \\
\hline$t(\mathrm{~nm})$ & $389(3)$ & $766(3)$ \\
$\varepsilon_{\infty, \mathrm{ZB}}$ & $6.8(0.3)$ & $7.2(0.3)$ \\
$\omega_{\mathrm{TO}, \mathrm{ZB}}\left(\mathrm{cm}^{-1}\right)$ & $466.5(0.5)$ & $465(1)$ \\
$\omega_{\mathrm{LO}, \mathrm{ZB}}\left(\mathrm{cm}^{-1}\right)$ & $590^{\mathrm{a}}$ & $590^{\mathrm{a}}$ \\
$\gamma_{\mathrm{ZB}}\left(\mathrm{cm}^{-1}\right)$ & $17(1)$ & $9(1)$ \\
$\varepsilon_{\infty, \|}$ & $7^{\mathrm{a}}$ & $6.8(0.2)$ \\
$\omega_{\mathrm{TO}, \|}\left(\mathrm{cm}^{-1}\right)$ & $464(1)$ & $457(1)$ \\
$\omega_{\mathrm{LO}, \|}\left(\mathrm{cm}^{-1}\right)$ & $590(10)$ & $590(10)$ \\
$\gamma_{\|}\left(\mathrm{cm}^{-1}\right)$ & $6(1)$ & $13(1)$ \\
$\varepsilon_{\infty, \perp}$ & $7^{\mathrm{a}}$ & $7^{\mathrm{a}}$ \\
$\omega_{\mathrm{TO}, \perp}\left(\mathrm{cm}^{-1}\right)$ & $477(1)$ & $476(1)$ \\
$\omega_{\mathrm{LO}, \perp}\left(\mathrm{cm}^{-1}\right)$ & $606(10)$ & $606(10)$ \\
$\gamma_{\perp}\left(\mathrm{cm}^{-1}\right)$ & $6(1)$ & $5(1)$ \\
$N\left(\mathrm{~cm}^{-3}\right)$ & $7.9(0.1) \times 10^{18}$ & $3.7(0.1) \times 10^{18}$ \\
$\mu\left[\mathrm{cm}^{2} /\left(\mathrm{Vs}^{1}\right)\right]$ & $125(10)$ & $400(10)$ \\
$N_{s}\left(\mathrm{~cm}^{-2}\right)$ & $8.0(4.8) \times 10^{13}$ & - \\
\hline \hline
\end{tabular}

${ }^{a}$ Assumed value, fixed during GIRSE data analysis.

maps associated with the WZ phonons are simply shifted by $90^{\circ}$ with respect to those of $100 \% \mathrm{WZ}$ (1011) domain A (see Fig. 5). A pronounced effect on all maps in the range of the sapphire phonon bands can also be observed. The corresponding spectral features for domain B show mirror azimuthal symmetry compared to the respective maps for domain A. The Müller matrix azimuthal maps for a film containing $100 \% \mathrm{WZ}$ (10)11) domains $\mathrm{C}$ and D are identical to those of domains A and $\mathrm{B}$, respectively. Consequently, for films containing equal fractions of WZ domains $\mathrm{A}$ and $\mathrm{C}$ or $\mathrm{B}$ and $\mathrm{D}$, the GIRSE data set would not be able to distinguish between the domains rotated with respect to each other by $180^{\circ}$. In this case, the offdiagonal elements of the dielectric tensor of the mixed-phase thin film become zero and the on-diagonal elements possess sensitivity only to the sum of the volume fractions of oppositely located domains. Likewise, whenever these domains have different volume fractions distinct features would occur in the GIRSE azimuthal maps for the off-diagonal-block elements of the Müller matrix $\left(\mathrm{M}_{13}, \mathrm{M}_{14}, \mathrm{M}_{23}\right.$, etc.). These maps in particular then allow differentiation of WZ domains with different volume fractions, and determination of their fraction parameters.

Figures 5(c), 5(g), and 5(k) exemplify the azimuthal maps for a thin film containing equal fractions of WZ domain $\mathrm{A}$ and $\mathrm{ZB}$ domain. Clearly, these maps can be viewed as a superposition of the constituting maps of each domain weighted by their volume fractions. Therefore appropriate analysis of the GIRSE data acquired at different (at least three) azimuthal positions within a range of $180^{\circ}$ can unambiguously render the volume fractions and phonon properties of the respective domains.

A second example considers a mixed-phase thin film containing WZ domains $\mathrm{B}, \mathrm{C}$ and $\mathrm{D}$ with different volume fractions, negligible amount of domain $\mathrm{A}$ and a relatively small fraction of $18 \% \mathrm{ZB}(002)$ domain [Figs. 5(d), 5(h), and 5(1)]. The azimuthal maps were calculated using fraction parameters obtained from our best-model calculation of sample II (see Table I). The presence of significant fractions of neighboring WZ domains creates a quasicontinuous pattern in the vicinity of the $\omega_{\mathrm{TO}, \perp}$ in the $\mathrm{M}_{12}$ map [Fig. 5(d)]. The ZB domain also creates unique fingerprints in $M_{12}$ close to the $\mathrm{ZB}$ TO frequency. Distinct spectral features occur in the offdiagonal-block element azimuthal maps [e.g., $\mathbf{M}_{13}$ and $\mathrm{M}_{43}$ in Figs. 5(h) and 5(1)] as a result of the differences in the volume fractions of oppositely located domains. Note that additional changes in the bands of total reflection of $p$ and $s$ polarized light also occur in the other Müller matrix elements. Thus the complete set of GIRSE data measured at different azimuths and angles of incidence provide sufficient sensitivity to uniquely pin point the polytype volume fraction and domain distribution. The above described model also allows the assessment of the phonon parameters of the two InN polytypes.

The sensitivity of the method to determine the polytype volume fraction is estimated to $\pm 2 \%$ for the given example when a pair of corresponding sets of TO, LO phonon mode frequencies (e.g., for polarizations along certain lattice axes of different polytypes) is separated from each other by at least the wave-number amount of their respective broadening parameters. Similar sensitivity to the WZ domain distribution can also be achieved if at least five measurements are performed at different azimuth angles within a range of $180^{\circ}$. The uncertainties are based on (i) the experimentally determined uncertainties on the GIRSE raw data (which enter the best-match model data analysis) and (ii) the differences in phonon mode signatures between different phases in general. For the general case, a practical limit of uncertainty is difficult to obtain, however, the present case of InN may serve as a good estimate for situations of similar mixtures of phases with substantially different phonon mode signatures. As substantially different we can define when a set (TO-LO)/anisotropic pair of sets of TO-LO phonon modes is separated from each other by at least the wave-number amount of their broadening parameters.

\section{GIRSE data analysis}

Figure 6 depicts selected experimental and best-match model calculated GIRSE data sets from the two samples discussed in this work. Different data sets are displayed for four sample azimuth positions. For convenience, vertical lines indicate the frequencies of the $\mathrm{WZ}$ InN phonon modes of $\mathrm{A}_{1}[\mathrm{w}-\operatorname{InN}(p)]$ and $\mathrm{E}_{1}[\mathrm{w}-\mathrm{InN}(s)]$ symmetry and ZB InN phonon modes (c-InN), in addition to the $A_{2 u}$ and $E_{u}$ phonon modes of sapphire (see Fig. 6). The Müller matrix element $\mathrm{M}_{12}$ belongs to the on-diagonal block elements, which are generally nonzero regardless of sample anisotropy. The off-diagonal block element $\mathrm{M}_{43}$ is zero for isotropic samples. Both elements 

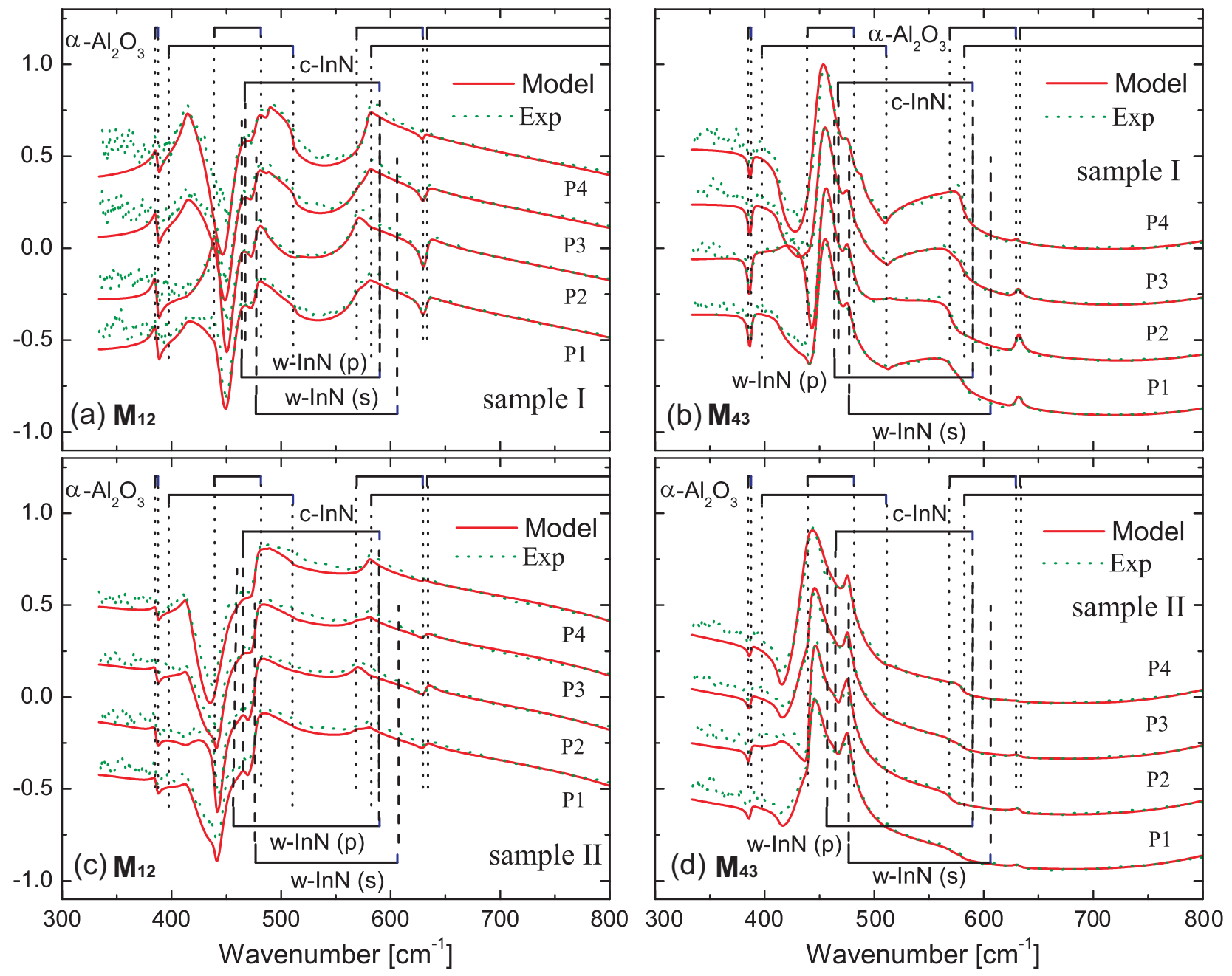

FIG. 6. (Color online) (a) Experimental (dotted lines) and best-model calculated (solid lines) GIRSE M $\mathrm{M}_{12}$ spectra of a mixed-phase InN thin film on $r$-plane sapphire (sample I) for different sample azimuth angles $\varphi$ between the plane of incidence and the plane perpendicular to the $r$-plane sapphire surface that contains the sapphire $c$ axis. Müller matrix spectra $\mathrm{M}_{12}$ correspond to in-plane azimuth positions $\varphi=-40.1^{\circ}(\mathrm{P} 1)$, $4.9^{\circ}(\mathrm{P} 2), 49.9^{\circ}(\mathrm{P} 3)$, and $94.8^{\circ}(\mathrm{P} 4)$. The frequencies of the wurtzite $\mathrm{InN}$ phonon modes of $\mathrm{A}_{1}(p)$ and $\mathrm{E}_{1}(s)$ symmetry and $\mathrm{ZB}$ InN phonon modes are indicated by vertical dashed lines. The $\mathrm{A}_{2 \mathrm{u}}$ and $\mathrm{E}_{\mathrm{u}}$ phonon modes of sapphire are indicated for clarity; (b) the same as in (a) for $\mathrm{M}_{43}$ of sample I, (c) the same as in (a) for $\mathrm{M}_{12}$ of sample II. Spectra correspond to $\varphi=-42.9^{\circ}(\mathrm{P} 1), 2.1^{\circ}(\mathrm{P} 2), 47.1^{\circ}$ (P3), and 92.1 ${ }^{\circ}$ (P4), and (d) the same as (c) for $\mathrm{M}_{43}$ of sample II. Data are shifted for clarity by 0.3 (P2), 0.6 (P2), and 0.9 (P4) in (a)-(d).

are chosen here exemplarily, and for brevity. The full available data set, however, was included during data analysis. As seen in Fig. 6, the spectral signatures in the vicinity of the indicated phonon modes change with sample rotation, and reveal their origin as being either caused by the substrate or the mixed-phase InN layer. Phonon signatures due to $\mathrm{A}_{1}$ and $\mathrm{E}_{1} \mathrm{WZ}$ InN modes occur. Subtle changes in the spectra in between the $A_{1}$ and $E_{1} W Z T O$ and $A_{1}$ and $E_{1}$ WZ LO phonon modes can be identified (Fig. 6). These changes are caused by the existence of the ZB phonon mode contributions within the mixed-phase InN layer as discussed in Sec. IV B.

The best-match model calculated data in Fig. 6 were obtained using the stratified layer model discussed in Sec. IV B, and the effective dielectric tensor for the mixed-phase $\mathrm{InN}$ layer described in Sec. III B 1. The best-match model calculations are performed by allowing to vary the $\mathrm{WZ} \mathrm{InN}$ parameters $\omega_{\mathrm{TO}, \|}, \gamma_{\|}, \omega_{\mathrm{TO}, \perp}, \gamma_{\perp}, N$ and $\mu$ as well as all ZB InN model dielectric function parameters. The WZ InN parameter $\varepsilon_{\text {inf }, \perp}=\varepsilon_{\text {inf }, \|}=7.04$ is taken from Ref. [36]. The $\mathrm{ZB} \mathrm{InN} \mathrm{parameter} \omega_{\mathrm{LO}}=590 \mathrm{~cm}^{-1}$ is taken as the average between the WZ LO mode parameters, and was fixed during analysis because the present data set is not sensitive to determine this parameter independently. In addition, all fraction parameters are freely adjustable, as well as the film thickness and the initial azimuth orientation parameter. The best-match model parameters are summarized in Table I. In addition, the dielectric function, phonon and free-charge carrier parameters of $\mathrm{ZB} \mathrm{InN} \mathrm{are} \mathrm{compared} \mathrm{to} \mathrm{previously}$ published results in Table IV.

\section{Polytype distribution and volume fraction}

The XRD pole figure measurements (Fig. 2) reveal that the WZ InN domains form at all four equivalent $\mathrm{ZB} \operatorname{InN}$ (111) planes. However, the WZ InN (1011) poles have different diffraction intensities (see Fig. 2), which indicates that the 
TABLE II. Structural factors $F$, linear absorption coefficients $\mu_{m}$, diffraction angles $\theta$, and unit cell volume $V_{\mathrm{uc}}$, of the respective ZB and $\mathrm{WZ}$ InN domains used in the calculation of the volume fraction. $F$ is calculated using the software CaRine Crystallography 3.1., $\mu_{m}$ and $\theta$ are taken from XRD database, and $V_{\mathrm{uc}}$ is estimated using the strain-free lattice parameters reported in Ref. [38].

\begin{tabular}{lccc}
\hline \hline & ZB(002) & WZ(1011) & WZ(0002) \\
\hline$|F|^{2}$ & 16786.56 & 3885.48 & 6500.18 \\
$\mu\left(\mathrm{cm}^{2} / \mathrm{g}\right)$ & $1.4 \times 10^{5}$ & $2.8 \times 10^{5}$ & $2.8 \times 10^{5}$ \\
$\theta($ degree $)$ & 18.02 & 16.67 & 15.80 \\
$V_{\text {uc }}\left(\AA^{3}\right)$ & 123.506 & 61.81 & 61.81 \\
\hline \hline
\end{tabular}

domains have different volume fractions. Previously, the formation of only one WZ domain in ZB InN (002) has been reported [37]. Recently, we have shown that in a semipolar WZ InN (1011) films only two of the four domains developed during growth [18]. These differences can be attributed to specific nucleation and growth conditions employed.

Since at a particular angle of inclination $\left(0^{\circ}\right.$ or $\left.7^{\circ}\right)$ and azimuth, only one peak related to $\mathrm{WZ}$ InN appears in the $2 \theta-\omega$ scans, each of the four WZ (1011) domains was treated as a domain with separate crystallographic orientation in the volume fraction analysis. The appropriate evaluation of the volume fraction of the $\mathrm{WZ}$ and $\mathrm{ZB}$ polymorphs requires the measurement of the $2 \theta-\omega$ integrated intensity for each of the $\mathrm{WZ}$ and $\mathrm{ZB}$ domains. Equation (5) is used to estimate the fraction of the four WZ InN (1011) domains, the WZ InN (0001) and the ZB (001) fractions. The parameters used in the calculation are listed in Table II and the respective volume fractions for the two samples are given in Table III. Note that the volume fraction of a given polymorph is inversely proportional to the squared structural factor of the specific plane [see Eq. (5)]. The structural factor of $\mathrm{ZB} \mathrm{InN} \mathrm{(002)} \mathrm{is}$ significantly larger that the structural factors of the WZ InN (1011) and (0002) planes (see Table II). Therefore a direct comparison of intensities would result in erroneous, largely overestimated values of the $\mathrm{ZB}$ InN fraction.

The volume fractions of $\mathrm{WZ}$ and $\mathrm{ZB}$ InN determined from GIRSE and XRD (Table III) show excellent agreement. According to the XRD results (Table III) the WZ InN (10i11) in sample I grows predominantly in two adjacent domains B $(21 \%)$ and C $(14 \%)$, while the growth of the other two domains, A and D is suppressed, which agrees very well with the GIRSE results. In sample II, the volume fraction distribution is different. Two of the WZ (1011) domains have significant volume fraction of $26 \%$ : $\mathrm{C}$ and $\mathrm{D}$, while the other two domains have smaller but still appreciable volume fractions of $10 \%$ (A) and $18 \%$ (B). The distribution of the WZ
(101) domains determined from GIRSE in this case shows some differences compared to XRD. We note that in GIRSE an area of $\approx 8 \times 8 \mathrm{~mm}^{2}$ is probed, while in XRD the beam spot was $1-\mathrm{mm}$ wide and $8-\mathrm{mm}$ long, which may partly explain the differences observed between the two methods. Additional uncertainty comes from the fact that the total fractions of domains $\mathrm{A}+\mathrm{C}$ and $\mathrm{B}+\mathrm{D}$ are very similar. This uncertainty may be reduced by measuring at a larger number of azimuth angles.

As discussed earlier the formation of WZ InN (0001) on top of the four equivalent $\mathrm{ZB} \mathrm{InN}\{111\}$ planes (Fig. 3) occur as a result of stacking fault and gives rise to the WZ InN inclusions. Although the four ZB InN $\{111\}$ planes are crystallographically identical, their polarity is different. Two adjacent ZB InN $\{111\}$ planes, rotated with respect to each other by $90^{\circ}$ are commonly labeled (111)A and (111)B, and have In and $\mathrm{N}$ polarity, respectively. Consequently, the $\mathrm{WZ} \mathrm{InN} \mathrm{will} \mathrm{grow}$ on the adjacent faces also with different polarities: (0001) (In-polar) WZ InN will grow on ZB InN (111)A and (0001) (Npolar) WZ InN will grow on ZB InN(111)B (see Figs. 3 and 1) [39]. Our results clearly show that WZ InN with both polarities occur with no preference, which indicates that the polarity does not play a determining role for the formation and growth rate of the WZ InN fraction. In contrast, in WZ InN (1011), we found that only one domain is dominant [18]. In this case the growth of WZ InN is stabilized on the ZB nucleation centers at an early stage during growth, and no macroscopic $\mathrm{ZB}$ InN polymorph was detected by XRD. For InN with predominant ZB structure, the formation of a single wurtzite (10 $\overline{1} 1$ ) domain (near the film surface) was previously reported [15]. In this latter case, the authors argued, based on XRD measurements, that initially the $\mathrm{ZB}$ InN growth occurs, and then WZ polymorph appears as defect during the proceeding growth. In order to gain further insight into the formation and development of the $\mathrm{ZB}$ and $\mathrm{WZ}$ domains in our samples, we performed cross-sectional TEM.

The TEM cross-section images reveal the presence of $\mathrm{ZB}$ InN and $\mathrm{WZ}$ InN in the two samples, in agreement with the XRD results (see Fig. 7). The $\langle 0001\rangle$ directions of the WZ domains include angles of $\approx 54^{\circ}$ with respect to the surface, which coincides with the angle between the $\langle 111\rangle$ and [001] of $\mathrm{ZB}$ InN. This result confirms that the WZ inclusions indeed form on the $\mathrm{ZB}$ InN $\langle 111\rangle$ as indicated by the XRD pole figure measurements, and as illustrated in Fig. 3. The observed slight spread in the values of the inclination angle of the WZ domains could be associated with a different tilt of the initial ZB (002) islands, on which the WZ polymorph forms. High-resolution TEM (HRTEM) [see Figs. 8(c) and 9(c)] shows that ZB InN islands form at the interface to the substrate in both films. These islands grow with their [002] axis parallel to the growth direction, forming $\langle 111\rangle$ facets. It is seen from Figs. 8(c)

TABLE III. Volume fractions of ZB and WZ InN polymorphs estimated by XRD and GIRSE analysis. The $a$-lattice parameter of the ZB InN and the $c$-lattice parameter of the WZ InN determined by XRD and HRTEM, respectively, are also listed.

\begin{tabular}{|c|c|c|c|c|c|c|c|c|c|}
\hline \multirow[b]{2}{*}{ Sample } & \multicolumn{7}{|c|}{ Volume fraction $(\%)$} & \multirow[b]{2}{*}{$a_{\mathrm{ZB}}(\AA)$} & \multirow[b]{2}{*}{$c_{\mathrm{WZ}}(\AA)$} \\
\hline & Method & $\mathrm{ZB}$ & WZ A & WZ B & WZ C & WZ D & WZ (0002) & & \\
\hline 1 & XRD & 55 & 2 & 21 & 14 & 2 & 6 & 5.00 & 5.73 \\
\hline 1 & IRSE & 57 & 0 & 23 & 20 & 0 & 0 & - & - \\
\hline 2 & XRD & 20 & 10 & 18 & 26 & 26 & 0 & 4.99 & 5.69 \\
\hline 2 & IRSE & 18 & 1 & 13 & 44 & 24 & 0 & - & - \\
\hline
\end{tabular}



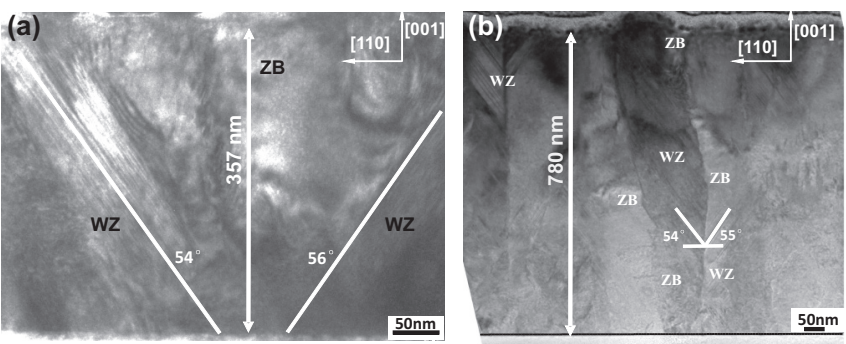

FIG. 7. TEM cross-sectional images of sample I (a) and sample II (b). Note the different scales in (a) and (b).

and 9(c) that WZ inclusions with their $\langle 0001\rangle$ parallel to the $\mathrm{ZB}\langle 111\rangle$ form already at this early stage of the growth. It is apparent, however, that the WZ crystal structure is not stabilized (as one would expect) and $\mathrm{ZB} \mathrm{InN} \mathrm{also} \mathrm{forms}$ on top of the WZ inclusions. Indeed, the HRTEM images taken from the middle parts of the films [Figs. 8(b) and 9(b)] reveal the presence of both WZ and ZB polymorphs. Either of the polymorphs may be dominant, however, stacking faults with varying densities are present within. It is seen from the HRTEM taken from the top of sample I that the ZB is the predominant polyomorph at the film surface [Fig. 8(a)]. A closer look at the surface area in the low-resolution TEM [Fig. 7(a)] reveals that ZB InN overgrows the WZ domains. A higher growth rate along the $\mathrm{ZB} \operatorname{InN}$ [001] than along WZ InN [0001] may explain this result. In contrast, as the film thickness increases the WZ polymorph becomes dominant in sample II. Figure 9(a) shows a HRTEM image taken from the only surface area where ZB InN could be detected. Even in this case, the WZ polyomorph covers the surface entirely. We note that previous XRD studies on similar InN samples indicated that the WZ polymorph is stabilized at the surface [15]. Sample II is grown at a higher temperature than sample I and it is also thicker by a factor of two. The higher growth temperature increases adatom mobility and facilitates the stabilization of the WZ polymorph, which becomes dominant with increasing film thickness. These findings have important implications for the electronic properties of ZB InN, in particular for correct determination of electron accumulation that occurs at the InN film surfaces. It is obvious that even in samples with predominant or similar ZB InN volume fractions, the surface may be covered by either of the polymorphs depending on the growth conditions.
Furthermore, an "M"-shape behavior of the ZB InN (002) RCs with minima for azimuth parallel to the sapphire $c$ axis and maxima for the perpendicular directions was found for both sample I and II. However, sample I exhibits stronger anisotropy with the RC width in the direction perpendicular to the $c$ axis being significantly higher than in sample II. The larger RC curve width in sample I can be associated with a mosaic tilt [see Fig. 2(a)]. For comparison, sample II does not show any tilt [see Fig. 2(b)], which results in much lower RC anisotropy. The lattice parameters of the $\mathrm{ZB} \mathrm{InN}$ are determined from XRD to be 5.00 and $4.99 \AA$ for films I and II, which are consistent with previously reported values $[15,16,40]$. A comparison to the previously reported lattice parameters of $\mathrm{ZB} \mathrm{InN} \mathrm{is} \mathrm{presented}$ in Table IV. The $c$-lattice parameters of the WZ domains in the two samples were estimated from TEM to be 5.73 and $5.69 \AA$ A for films I and II, respectively. The values for the films thickness determined by GIRSE (Table I) agree very well with the respective values estimated from TEM (see Fig. 7).

\section{E. Phonon and free-charge carrier properties}

The ZB TO frequency is determined to be $466.5 \pm 1$ and $465.0 \pm 1 \mathrm{~cm}^{-1}$ for sample I and II, respectively (Table I). Due to the complex structure of the films and the lack of reference strain-free lattice parameters of $\mathrm{ZB} \mathrm{InN}$, it was not possible to evaluate the strain in the films from XRD. However, since film II is much thicker one can speculate that it is more relaxed. This would be consistent with the observed slight redshift of the TO phonon frequency between the two samples. Earlier works on $\mathrm{ZB}$ InN grown on GaAs using InAs buffer layer determined the TO frequency from Raman measurements to be $457 \mathrm{~cm}^{-1}$ [41] (see Table IV). This value is significantly redshifted with respect to TO frequencies determined for our film. The lower lattice parameter of the InN film from Ref. [41] (4.97 $\AA$ ) compared to the lattice parameters of films I and II studied here, may explain to a certain extent the observed differences (Table IV). However, more factors such as growth mode, carrier concentration and defect distribution may also affect the TO frequencies. More recent work on $\mathrm{ZB}$ InN grown on $3 \mathrm{C}-\mathrm{SiC}$ reported $\mathrm{TO}$ frequencies from infrared ellipsometry in the range $468.9-470.0 \mathrm{~cm}^{-1}$ depending on growth temperature and film thickness (see Table IV) [40]. These samples also contained small fractions of WZ polymorph while no details were reported on how the volume fractions (up to 11\%) were
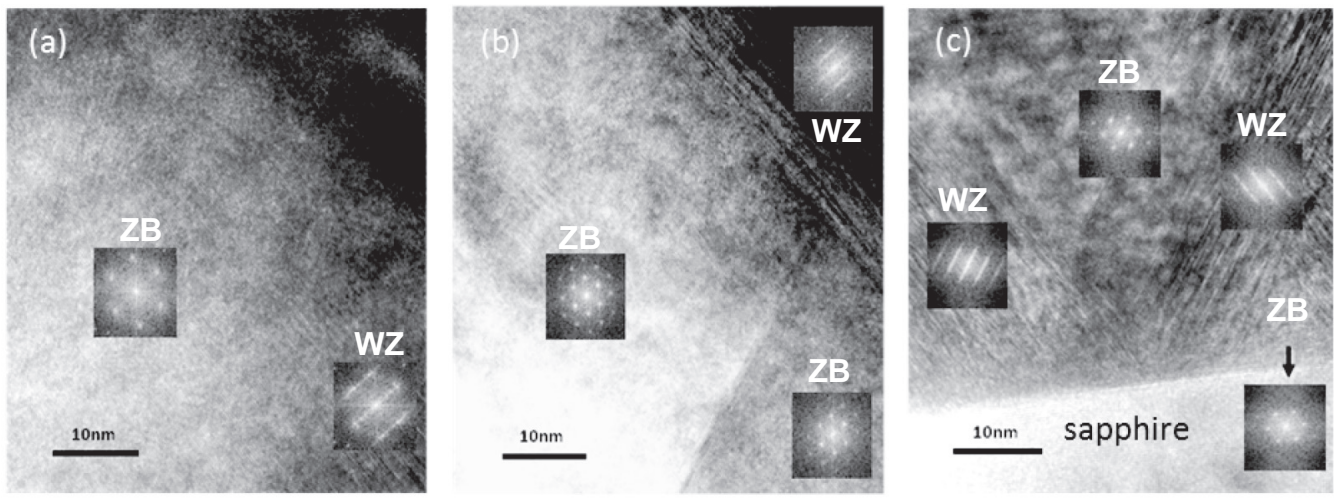

FIG. 8. HRTEM cross-sectional images acquired along the thickness of sample I and the respective FFT patterns: (a) surface, (b) interior, and (c) interface to the substrate. 

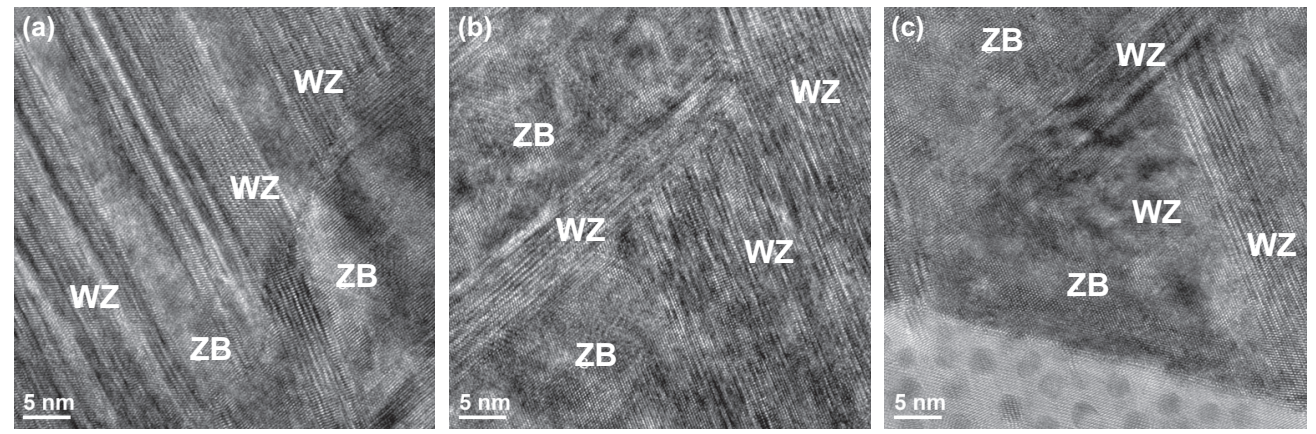

FIG. 9. HRTEM cross-sectional images acquired along the thickness of sample II: (a) surface, (b) interior, and (c) interface to the substrate.

determined. However, we note that the presence of WZ InN polyomorph has not been accounted for in the IRSE analysis in Ref. [40]. The strain-free $\mathrm{E}_{1}(\mathrm{TO})$ frequency of $\mathrm{WZ} \mathrm{InN} \mathrm{is}$ $477.9 \mathrm{~cm}^{-1}$ [36] and if the WZ inclusions are note accounted for this might affect the accuracy of the ZB TO phonon frequency. The lattice parameters of the $\mathrm{ZB} \mathrm{InN}$ films on 3C-SiC are reported to be $5.01 \AA$ [40], which is consistent with the observed blue shift of TO frequencies in these samples with respect to our result (see Table IV).

Lattice strain and crystal defects typically broaden the TO phonon resonances. Therefore higher degree of strain and mosaicity can explain the observed larger phonon broadening in sample I compared to sample II (see Table I). This agrees well with the higher RC broadening and anisotropy in sample I found by XRD and it is also consistent with our speculation for higher degree of strain in the film.

The high-frequency dielectric function model parameter of $\mathrm{ZB} \mathrm{InN}, \varepsilon_{\infty, \mathrm{ZB}}$ is determined to be $6.8 \pm 0.3$ and $7.2 \pm 0.3$ for samples I and II, respectively, in good agreement with previous results (see Table IV) [40]. We also note that the InN film exhibiting higher free electron concentration, sample I, has a smaller value of $\varepsilon_{\infty, \mathrm{ZB}}$ (see Table I). A similar trend was also observed previously for films with higher electron concentrations compared to those in samples I and II [40]. In addition, we have estimated the static dielectric constant parameters of $\mathrm{ZB} \mathrm{InN,} \varepsilon_{0, \mathrm{ZB}}$ of $10.9-11.6$, by using the best-match model derived TO phonon mode frequency and high-frequency dielectric constant parameters and applying the Lydanne-Sachs-Teller relation (see Table IV).

The $\omega_{\mathrm{TO}, \perp}\left[\mathrm{E}_{1}(\mathrm{TO})\right]$ phonon frequencies of the $\mathrm{WZ} \mathrm{InN}$ inclusions in the two samples are redshifted with respect to the strain-free value of $477.9 \mathrm{~cm}^{-1}$ [36] (see Table I). The shift of the $\mathrm{E}_{1}(\mathrm{TO})$ frequency is given by

$$
\begin{aligned}
\Delta \omega_{E_{1}}= & a_{E_{1}}\left(\epsilon_{x x}+\epsilon_{y y}\right)+b_{E_{1}} \epsilon_{z z} \\
& \pm c\left(E_{E_{1,2}}\left[\left(\epsilon_{x x}-\epsilon_{y y}\right)^{2}+4 \epsilon_{x y}^{2}\right]^{1 / 2} .\right.
\end{aligned}
$$

The coefficients, $a_{j}, b_{j}$, and $c_{j}$ are the corresponding mode deformation potentials per unit strain, and $\epsilon_{x x}, \epsilon_{y y}$, and $\epsilon_{z z}$ are the normal strains along the WZ InN [112̄0], [1100], and [0001] directions, respectively. For group-III nitrides, the phonon deformation potentials are negative and the $c_{j}$ deformation potential is typically smaller than $a_{j}, b_{j}$ [21]. The $a_{j}$ and $b_{j}$ deformation potentials in InN have been previously determined [36] but the $c_{j}$ remains unknown. In our GIRSE analyses, no anisotropy of the $E_{1}(\mathrm{TO})$ phonon along the two perpendicular directions in the basal plane is considered, and the results for the $\omega_{\mathrm{TO}, \perp}$ represents an isotropically averaged value, hence the last term in Eq. (25) can be neglected. We note that in group-III nitride films grown on off-axis oriented sapphire substrates the strain in the basal plane is anisotropic $\left(\epsilon_{x x} \neq \epsilon_{y y}\right)$ and has more complex behavior (see [21,42]). For (1011)-plane WZ InN grown pseudomorphycally on GaN $\epsilon_{x x}$ and $\epsilon_{z z}$ have positive values, while $\epsilon_{y y}$ is negative with $\left|\epsilon_{x x}\right| \ll\left|\epsilon_{y y}\right|$ and $\epsilon_{x y}$ is zero. This would result in a blueshift of the $E_{1}(\mathrm{TO})$ phonon. However, our films are grown directly on sapphire and the normal strains may differ from the pseudomorphic growth situation. Indeed, the $c$-lattice parameter of the WZ InN inclusions estimated from TEM is larger than the strain-free value of $5.7002 \AA$ [38] for sample I and it is smaller for sample II. Thus the strain along the $z$ direction is tensile for sample I but it becomes compressive for sample II. The relationship between the normal strains in

TABLE IV. Structural, phonon, dielectric and free-charge carrier parameters of ZB InN in comparison with previously published experimental results: TO phonon frequency $\omega_{\mathrm{TO}, \mathrm{ZB}}$, high-frequency dielectric function limit $\varepsilon_{\infty, \mathrm{ZB}}$, static dielectric constant $\varepsilon_{0, \mathrm{ZB}}, N$ and $\mu$ are the bulk free electron concentration and mobility, respectively, $N_{s}$ is the electron accumulation density at the surface, and $a_{\mathrm{ZB}}$ is the

\begin{tabular}{|c|c|c|c|c|c|c|c|}
\hline & $\omega_{\mathrm{TO}, \mathrm{ZB}}\left(\mathrm{cm}^{-1}\right)$ & $\varepsilon_{\infty, \mathrm{ZB}}$ & $\varepsilon_{0, \mathrm{ZB}}$ & $N\left(\mathrm{~cm}^{-3}\right)$ & $\mu\left[\mathrm{cm}^{2} /(\mathrm{Vs})\right]$ & $N_{s}\left(\mathrm{~cm}^{-2}\right)$ & $a_{\mathrm{ZB}}(\AA)$ \\
\hline this work & $465.0-466.5$ & $6.8-7.2$ & $10.9-11.6$ & $3.7-7.2 \times 10^{18}$ & $125-400$ & $8.0 \times 10^{13}$ & $4.99-5.00$ \\
\hline Literature & $\begin{array}{c}468.9-470.0^{\mathrm{a}} \\
457^{\mathrm{b}}\end{array}$ & $6.64-6.97^{\mathrm{a}}$ & & $\begin{array}{r}2.1-3.8 \times 10^{19, \mathrm{a}} \\
3.2 \times 10^{19, \mathrm{c}}\end{array}$ & $580-835^{a}$ & $9.1 \times 10^{12, c}$ & $\begin{array}{c}5.01^{\mathrm{a}} \\
4.97,^{\mathrm{b}} 4.986^{\mathrm{d}}\end{array}$ \\
\hline
\end{tabular}
lattice parameter.

\footnotetext{
${ }^{\text {a Reference [40]. }}$

${ }^{\mathrm{b}}$ Reference [41].

${ }^{\mathrm{c} R e f e r e n c e ~[27] . ~}$

${ }^{\mathrm{d}}$ Reference [15].
} 
nitride films with an arbitrary surface orientations is given by [42]

$$
A_{z} \epsilon_{z z}=A_{x} \epsilon_{x x}+A_{y} \epsilon_{y y},
$$

where

$$
\begin{aligned}
& A_{x}=C_{12}-C_{13}-\left(C_{12}+C_{13}\right) \cos 2 \vartheta, \\
& A_{y}=C_{11}-C_{13}-\left(C_{11}+C_{13}\right) \cos 2 \vartheta, \\
& A_{z}=C_{33}-C_{13}+\left(C_{13}+C_{33}\right) \cos 2 \vartheta
\end{aligned}
$$

are the biaxial relaxation coefficients

The biaxial strain coefficients $\mathrm{A}_{j}$, and $\vartheta=54^{\circ}$ are the same for samples I and II. Thus the different type of strain along the $\mathrm{z}$ directions in the two films indicate different type of distortion of the basal (0001) plane.

The shift of the $A_{1}(\mathrm{TO})$ or $\omega_{\mathrm{TO},||}$ frequency with respect to the strain-free value is given by

$$
\Delta \omega_{A_{1}}=a_{A_{1}}\left(\epsilon_{x x}+\epsilon_{y y}\right)+b_{A_{1}} \epsilon_{z z} .
$$

It should be noted that the redshift between the $E_{1}(\mathrm{TO})$ frequencies of sample I and II is much smaller than the respective difference regarding the $A_{1}(\mathrm{TO})$ frequency. This can be partly related to different deformation potentials of the $E_{1}(\mathrm{TO})$ and $A_{1}(\mathrm{TO})$ phonons. However, most probably the last term of Eq. (25) is significant and could not be neglected. A detailed study of the strain in the films combined with polarized Raman scattering measurements would shed light on this issue and will be reported elsewhere.

The free electron concentration is found to be $7.9 \times$ $10^{18} \mathrm{~cm}^{-3}$ (sample I) and $3.7 \times 10^{18} \mathrm{~cm}^{-3}$ (sample II), which is significantly lower than the concentrations previously observed in ZB InN [16,40] (see Table IV). It is interesting to point out that these carrier concentrations are comparable or even lower than the typical free electron concentrations measured in nonpolar InN films $[23,43,44]$. The mobility in sample II is as high as $400 \mathrm{~cm}^{2} / \mathrm{Vs}$, which is comparable or even higher to the mobility parameters of nonpolar InN $[18,45,46]$. These observations are quite encouraging given the complex microstructure of the films. It is thus plausible to suggest that if $\mathrm{WZ}$ polymorph inclusion can be minimized the transport parameters of $\mathrm{ZB} \mathrm{InN}$ can be substantially improved, and thus the use of $\mathrm{ZB} \mathrm{InN}$ as an alternative material to nonpolar $\mathrm{InN}$ in future device applications can be justified.

It is also interesting to look at the surface electron properties since the ubiquitous surface electron accumulation has been so far reported to occur at all InN surfaces: WZ polar, nonpolar and semipolar, as well as at the ZB InN surface [23,26-28]. However, for the $\mathrm{ZB} \mathrm{InN}$, no information on the purity of the polymorph was given [27]. Our results show that the distribution of the polymorphs along film thickness may differ substantially depending on the growth conditions and thickness and must be carefully evaluated to avoid erroneous interpretations. A surface layer with electron accumulation density of $8.0 \times 10^{13} \mathrm{~cm}^{-2}$ is identified from the GIRSE data analysis in sample I. This value is somewhat higher than previously reported results (see Table IV). Note that sample I contains larger fraction of $\mathrm{ZB} \mathrm{InN}$ and its surface is covered entirely by the ZB polymorph only [see Fig. 8(a)]. In contrast, at the surface of sample II no electron accumulation was observed. In this case, however, the sample surface region contains predominantly the WZ polymorph [see Fig. 9(a)] but no particular WZ plane is parallel to the sample surface as the WZ InN (1011) plane is tilted by $7^{\circ}$. Our results suggest that an off-cut orientation may provide pathway to minimize or eliminate surface electron accumulation in $\mathrm{InN}$ heterostructures. The GIRSE results on the surface electron accumulation densities in the two samples are consistent with scanning tunneling spectroscopy measurements, which will be reported elsewhere.

\section{CONCLUSIONS}

We have presented a combined $\mathrm{x}$-ray diffraction and infrared ellipsometry analysis method to identify wurtizte and zinc-blende domains and to quantify their volume fraction parameters in thin mixed-phase epitaxial films. A linear homogenization approach considering each polymorph domain to be represented by an individual dielectric tensor weighted by its volume fraction is shown to provide excellent description of the infrared response of the mixed-phase films. We have shown that distinct changes in the GIRSE azimuthal maps of the mixed-phased thin films occur upon azimuth rotation and which depend on the polytype domain fraction and crystallographic orientation. This allows the determination of polytype volume fraction and domain distribution, as well as the assessment of the phonon parameters of $\mathrm{WZ}$ and $\mathrm{ZB}$ polytypes in mixed-phase epitaxial films. The sensitivity of the GIRSE method for volume fraction and domain distribution is estimated to $\pm 2 \%$ in the given examples and the limits of uncertainties in the general case are discussed.

We have applied our methods to a comprehensive study on structural and vibrational properties of mixed-phase $\mathrm{InN}$ epitaxial films containing ZB and WZ domains. The structural analysis has revealed that the $\mathrm{ZB} I n N$ nucleates at the interface with the $r$-plane sapphire and that WZ InN (0001) grows on top of the ZB InN $\{111\}$ faces at the early stage of the growth. However, if the growth temperature is kept sufficiently low the WZ polymorph is not stabilized and appears mainly as stacking faults. As a result, the surface region contains ZB polymorph only. On the other hand, at higher growth temperature the ZB volume fraction is significantly smaller and the film surface is covered mostly by WZ InN. The ZB TO phonon frequencies are determined to be $465-466 \mathrm{~cm}^{-1}$, the high-frequency limit parameters of the dielectric function are in the range of 6.8-7.2, and the static dielectric constant parameter is estimated to be 10.9-11.6. The WZ InN $E_{1} \mathrm{TO}$ and $\mathrm{A}_{1}(\mathrm{TO})$ frequency shifts indicate complex anisotropic distortion of the basal hexagonal plane due to the anisotropy of the substrate surface. The GIRSE analysis has indicated the presence of a distinct electron accumulation at the surface of the film with larger $\mathrm{ZB} \mathrm{InN} \mathrm{volume} \mathrm{fraction.} \mathrm{On} \mathrm{the} \mathrm{other} \mathrm{hand,} \mathrm{no} \mathrm{accumulation}$ of electrons could be inferred for the sample with low ZB InN content and surface regions containing WZ InN (1011) $7^{\circ}$ offcut orientation. Our results suggest that although surface accumulation occurs at the $\mathrm{ZB}$ surfaces its value may be tuned by the type of polymorph and surface orientation of the films. 


\section{ACKNOWLEDGMENTS}

We acknowledge support from the Swedish Research Council (VR) under grant No.2013-5580, the Swedish Governmental Agency for Innovation Systems (VINNOVA) under the VINNMER international qualification program, Grant No. 2011-03486, and the Swedish Foundation for Strategic
Research (SSF), under Grant No. FFL12-0181. The financial support by the Linköping Linnaeus Initiative on Nanoscale Functional Materials (LiLiNFM) supported by VR is gratefully acknowledged. The authors further acknowledge grant support by the National Science Foundation under awards MRSEC DMR-0820521, by the University of Nebraska-Lincoln, J. A. Woollam Co., Inc., and J. A. Woollam Foundation.
[1] H. Morkoç and S. N. Mohammad, Science 267, 51 (1995).

[2] F. A. Ponce and D. P. Bour, Nature (London) 386, 351 (1997).

[3] K. Okamoto, I. Niki, A. Shvartser, Y. Narukawa, T. Mukai, and A. Scherer, Nat. Mater. 3, 601 (2004).

[4] F. Qian, Y. Li, S. Gradečak, H. G. Park, Y. Dong, Y. Ding, Z. L. Wang, and C. M. Lieber, Nat. Mater. 7, 701 (2008).

[5] Y. Taniyasu, M. Kasu, and T. Makimoto, Nature (London) 441, 325 (2006)

[6] J. W. Schwede, I. Bargatin, D. C. Riley, B. E. Hardin, S. J. Rosenthal, Y. Sun, F. Schmitt, P. Pianetta, R. T. Howe, Z. X. Shen et al., Nat. Mater. 9, 762 (2010).

[7] K. Lischka, in Nitrides as Seen by the Technology, edited by T. Paskova and B. Monemar (Research Signpost, Trivandrum, India, 2002).

[8] J. Wu, J. Appl. Phys. 106, 011101 (2009).

[9] B. Daudin, G. Feuillet, J. Hübner, Y. Samson, F. Widmann, A. Philippe, C. Bru-Chevallier, G. Gillot, E. Bustarret, G. Bentoumi et al., J. Appl. Phys. 84, 2295 (1998).

[10] D. J. As, T. Frey, D. Schikora, K. Lischka, V. Cimalla, J. Pezoldt, R. Goldhahn, S. Kaiser, and W. Gebhardt, Appl. Phys. Lett. 76, 1686 (2000).

[11] D. G. Pacheco-Salazar, S. F. Li, F. Cerdeira, E. A. Meneses, J. R. Leite, L. M. R. Scolfaro, D. J. As, and K. Lischka, J. Cryst. Growth 284, 379 (2005).

[12] J. Schörmann, S. Potthast, D. J. As, and K. Lischka, Appl. Phys. Lett. 89, 131910 (2006).

[13] E. Monroy, M. Hermann, E. Sarigiannidou, T. Andreev, P. Holliger, S. Monnoye, H. Mank, B. Daudin, and M. Eickhoff, J. Appl. Phys. 96, 3709 (2004).

[14] M.-Y. Xie, N. Ben Sedrine, S. Schöche, H. Long, B. Monemar, T. Hofmann, M. Schubert, X. Wang, A. Yoshikawa, K. Wang et al., J. Appl. Phys. 115, 163504 (2014).

[15] V. Cimalla, J. Pezoldt, G. Ecke, R. Kosiba, O. Ambacher, L. Spieß, G. Teichert, H. Lu, and W. J. Schaff, Appl. Phys. Lett. 83, 3468 (2003).

[16] J. Schörmann, D. J. As, K. Lischka, P. Schley, R. Goldhahn, S. F. Li, W. Löffler, M. Hetterich, and H. Kalt, Appl. Phys. Lett. 89, 261903 (2006).

[17] C. L. Hsiao, T. W. Liu, C. T. Wu, H. C. Hsu, G. M. Hsu, L. C. Chen, W. Y. Shiao, C. C. Yang, A. Gällström, P.-O. Holtz et al., Appl. Phys. Lett. 92, 111914 (2008).

[18] V. Darakchieva, M. Y. Xie, N. Franco, F. Giuliani, B. Nunes, E. Alves, C. L. Hsiao, L. C. Chen, T. Yamaguchi, Y. Takagi et al., J. Appl. Phys. 108, 073529 (2010).

[19] S. Sanorpim, P. Jantawongrit, J. S. Kuntharin, C. Thanachayanont, T. Nakamura, R. Katayama, and K. Onabe, Phys. Status Solidi C 6, S376 (2009).

[20] V. Darakchieva, J. Birch, M. Schubert, T. Paskova, S. Tungasmita, G. Wagner, A. Kasic, and B. Monemar, Phys. Rev. B 70, 045411 (2004).
[21] V. Darakchieva, T. Paskova, M. Schubert, H. Arwin, P. P. Paskov, B. Monemar, D. Hommel, M. Heuken, J. Off, F. Scholz et al., Phys. Rev. B 75, 195217 (2007).

[22] V. Darakchieva, T. Paskova, and M. Schubert, in Nitrides with Nonpolar Surfaces, edited by T. Paskova (Wiley-VCH Verlag $\mathrm{GmbH} \& \mathrm{Co}, \mathrm{KGa}$, Weinheim, 2008).

[23] V. Darakchieva, M. Schubert, T. Hofmann, B. Monemar, C. L. Hsiao, T. W. Liu, L. C. Chen, W. J. Schaff, Y. Takagi, and Y. Nanishi, Appl. Phys. Lett. 95, 202103 (2009).

[24] M. Schubert, in Handbook of Ellipsometry, edited by H. Thompkins and E. A. Irene (William Andrew Publishing, Highland Mills, 2004).

[25] H. Fujiwara, Spectroscopic Ellipsometry (John Wiley \& Sons, New York, 2007).

[26] I. Mahboob, T. D. Veal, C. F. McConville, H. Lu, and W. J. Schaff, Phys. Rev. Lett. 92, 036804 (2004).

[27] P. D. C. King, T. D. Veal, C. F. McConville, F. Fuchs, J. Furthmüller, F. Bechstedt, P. Schley, R. Goldhahn, J. Schörmann, D. J. As et al., Appl. Phys. Lett. 91, 092101 (2007).

[28] S. Laziç, E. Gallardo, J. M. Calleja, F. Agulló-Rueda, J. Grandal, M. A. Sánchez-Garcia, E. Calleja, E. Luna, and A. Trampert, Phys. Rev. B 76, 205319 (2007).

[29] M. Schubert, Infrared Ellipsometry on Semiconductor Layer Structures: Phonons, Plasmons and Polaritons, Springer Tracts in Modern Physics Vol. 209 (Springer, New York, 2004).

[30] M. Schubert, T. E. Tiwald, and C. M. Herzinger, Phys. Rev. B 61, 8187 (2000).

[31] T. Hofmann, C. M. Herzinger, C. Krahmer, K. Streubel, and M. Schubert, Phys. Status Solidi A 205, 779 (2008).

[32] T. Hofmann, V. Darakchieva, B. Monemar, H. Lu, W. J. Schaff, and M. Schubert, J. Electron. Mater. 37, 611 (2008).

[33] T. Hofmann, P. Kühne, S. Schöche, J. T. Chen, U. Forsberg, E. Janzén, N. B. Sedrine, C. M. Herzinger, J. A. Woollam, M. Schubert et al., Appl. Phys. Lett. 101, 192102 (2012).

[34] S. Schöche, P. Kühne, T. Hofmann, M. Schubert, D. Nilsson, A. Kakanakova-Georgieva, E. Janzén, and V. Darakchieva, Appl. Phys. Lett. 103, 212107 (2013).

[35] M. Schubert, B. Rheinländer, E. Franke, H. Neumann, T. E. Tiwald, J. A. Woollam, J. Hahn, and F. Richter, Phys. Rev. B 56, 13306 (1997).

[36] V. Darakchieva, P. P. Paskov, E. Valcheva, T. Paskova, B. Monemar, M. Schubert, H. Lu, and W. J. Schaff, Appl. Phys. Lett. 84, 3636 (2004).

[37] V. Cimalla, V. Lebedev, F. M. Morales, R. Goldhahn, and O. Ambacher, Appl. Phys. Lett. 89, 172109 (2006).

[38] V. Darakchieva, M.-Y. Xie, F. Tasnádi, I. A. Abrikosov, L. Hultman, B. Monemar, J. Kamimura, and K. Kishino, Appl. Phys. Lett. 93, 261908 (2008). 
[39] A. Koukitu, Y. Kumagai, and H. Seki, in Nitrides as seen by the Technology, edited by T. Paskova and B. Monemar (Research Signpost, Trivandrum, India, 2002).

[40] P. Schley, R. Goldhahn, C. Napierala, G. Gobsch, J. Schörmann, D. J. As, K. Lischka, M. Feneberg, and K. Thonke, Semicond. Sci. Technol. 23, 055001 (2005).

[41] A. Tabata, A. P. Lima, L. K. Teles, L. M. R. Scolfaro, J. R. Leite, V. Lemos, B. Schöttker, T. Frey, D. Schikora, and K. Lischka, Appl. Phys. Lett. 74, 362 (1999).

[42] M.-Y. Xie, F. Tasnádi, I. A. Abrikosov, L. Hultman, and V. Darakchieva, Phys. Rev. B 86, 155310 (2012).
[43] G. Koblmüller, A. Hirai, F. Wu, C. S. Gallinat, G. D. Metcalfe, H. Shen, M. Wraback, and J. S. Speck, Appl. Phys. Lett. 93, 171902 (2008).

[44] G. Koblmüller, G. D. Metcalfe, M. Wraback, F. Wu, C. S Gallinat, and J. S. Speck, Appl. Phys. Lett. 94, 091905 (2009).

[45] H. Lu, W. J. Schaff, L. F. Eastmann, J. Wu, W. Walukiewicz, V. Cimalla, and O. Ambacher, Appl. Phys. Lett. 83, 1136 (2003).

[46] A. O. Ajagunna, E. Iliopoulos, G. Tsiakatouras, K. Tsagaraki, M. Androulidaki, and A. Georgakilas, J. Appl. Phys. 107, 024506 (2010) 